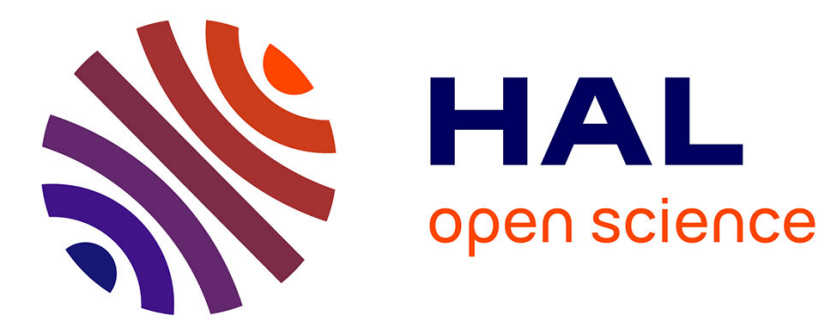

\title{
Multiplicative-error models with sample selection
}

Koen Jochmans

\section{To cite this version:}

Koen Jochmans. Multiplicative-error models with sample selection. 2014. hal-00987290

\section{HAL Id: hal-00987290 \\ https: / hal-sciencespo.archives-ouvertes.fr/hal-00987290}

Preprint submitted on 5 May 2014

HAL is a multi-disciplinary open access archive for the deposit and dissemination of scientific research documents, whether they are published or not. The documents may come from teaching and research institutions in France or abroad, or from public or private research centers.
L'archive ouverte pluridisciplinaire HAL, est destinée au dépôt et à la diffusion de documents scientifiques de niveau recherche, publiés ou non, émanant des établissements d'enseignement et de recherche français ou étrangers, des laboratoires publics ou privés.

\section{()ㅜ) $\Theta$}

Distributed under a Creative Commons Attribution - NoDerivatives| 4.0 International 


\section{Multiplicative-error models with sample selection}

Koen Jochmans

Sciences Po Economics Discussion Papers 


\title{
Multiplicative-error models with sample selection
}

\author{
Koen Jochmans $\dagger$ \\ Department of Economics, Sciences Po, Paris
}

[Revised February 28, 2014]

\begin{abstract}
This paper presents simple approaches to deal with sample selection in models with multiplicative errors. GMM estimators are constructed for both cross-section data and for panel data. These estimators build only on a specification of the conditional mean of the outcome of interest and are, therefore, semiparametric in nature. In particular, the distribution of unobservables is left unspecified. In the panel-data case, we further allow for group-specific fixed effects whose relation to covariates is left unrestricted. We derive distribution theory for both sampling situations and present Monte Carlo evidence on the finite-sample performance of the approach.
\end{abstract}

Keywords: nonlinear model; sample selection; semiparametric inference; two-stage estimation

\section{Introduction}

The detrimental effects of estimating economic models from non-randomly selected samples on statistical inference are well known. While the issue has received a substantial amount of attention in the literature, the proposed solutions have been confined mostly to the linear regression model; Gronau (1973) and Heckman (1974, 1978, 1979) have provided seminal contributions. However, sample selection is no less of a problem with nonlinear specifications, and the literature has been rather slow with devising flexible approaches to inference for such situations. ${ }^{1}$

This paper discusses relatively simple procedures to estimate nonlinear models with an additive- or multiplicative-error structure when the data is subject to sample selection. One leading example are models for count data. Such models are widely used in a variety of fields in economics (see Cameron and Trivedi 2006); Terza (1998), Winkelmann (1998), and Greene (2009) have given some attention to the issue of sample selection in such cases in a fully parametric setting. The approach taken here is semiparametric in the sense that it does not pin down the distribution of unobservables, and is applied both to models for cross-section data and to models for short panel data. We consider GMM estimators constructed from moment conditions that are inspired by a differencing argument introduced

$\dagger$ Address for correspondence: Sciences Po, Department of Economics, 28 rue des Saints-Pères, 75007 Paris, France. E-mail: koen.jochmans@sciencespo.fr.

\footnotetext{
${ }^{1}$ Of course, one may consider taking a full-information approach to inference, but specifying the full likelihood will require some tedious choices on the distribution of unobservables, and leads to estimators that are complicated to compute.
} 
by Chamberlain (1992) in a different context, present distribution theory, and report on the results from Monte Carlo experiments.

In the cross-sectional case, our proposal can be seen as a generalization of the classic contributions of Powell (1987) and Ahn and Powell (1993) on linear sample-selection models to nonlinear situations. Under rather conventional assumptions, our estimator converges at the parametric rate and has a limit distribution that is normal, with a variance that can be consistently estimated. A related generalization of the aforementioned works can be found in Blundell and Powell (2004). Their suggestion can equally be used to tackle sample selection, albeit in a different class of nonlinear models. The setup they consider and the one entertained here are not nested.

One nice feature of our strategy - contrary to, say, Blundell and Powell (2004) - is that it extends naturally to fixed-effect specifications for panel data. Consistent estimation of models with group-specific nuisance parameters is well-known to be problematic under asymptotics where the number of groups grows large while the number of observations per group remains fixed; see Arellano and Honoré (2001) and Lancaster (2000) for discussions and literature reviews. It is therefore not surprising that, besides focussing exclusively on linear models, the literature has favored a random-effect approach to inference in such cases; see, notably, Verbeek and Nijman (1992), Wooldridge (1995), and Rochina-Barrachina (2008). Only Kyriazidou $(1997,2001)$ has taken a fixed-effect perspective on the issue and, indeed, our proposal here can be interpreted as the corresponding version for models with multiplicative unobservables. Like in her case, the presence of between-group heterogeneity implies that our estimator will have a nonparametric convergence rate. Its asymptotic distribution remains normal, however, and asymptotically-valid inference can be performed using a plug-in estimator of the asymptotic variance.

In Section 2 below, we first deal with sample selection in a cross-sectional framework. In Section 3 we modify our approach to fixed-effect models for panel data. Proofs for both sections are collected in Appendix A and Appendix B, respectively.

\section{A semiparametric approach for cross-section data}

\subsection{The model and moment conditions}

For an integer $n$ and i.i.d. random variables $\left\{y_{i}, x_{i}, u_{i}\right\}_{i=1}^{n}$, consider the conditional-mean model

$$
\mathcal{E}\left[y_{i} \mid x_{i}, u_{i}\right]=\mu\left(x_{i} ; \alpha_{0}\right)+\varphi\left(x_{i} ; \beta_{0}\right) u_{i}
$$

where $\mu$ and $\varphi$ are functions that are known up to the Euclidean parameter $\theta_{0} \equiv\left(\alpha_{0}^{\prime}, \beta_{0}^{\prime}\right)^{\prime}$. Our aim will be to infer $\theta_{0}$ from a sample into which observations have self-selected. The selection process is modelled as a threshold-crossing model for the binary selection indicator $s_{i}$, with propensity score

$$
\operatorname{Pr}\left[s_{i}=1 \mid p_{i}\right]=\mathcal{E}\left[1\left\{p_{i} \geq v_{i}\right\} \mid p_{i}\right],
$$


for $p_{i}=p\left(z_{i}\right)$ an estimable aggregator mapping observables $z_{i}$ to the real line; $x_{i}$ and $z_{i}$ need not be disjoint. We view $\left(u_{i}, v_{i}\right)$ as representing unobserved heterogeneity that jointly influence $\left(y_{i}, s_{i}\right)$. These latent factors are taken to be independent of the observable characteristics $\left(x_{i}, z_{i}\right)$, but not necessarily of each other. The sample-selection problem, then, is to perform inference on $\theta_{0}$ from a random sample in which realizations of $\left(s_{i}, x_{i}, z_{i}\right)$ are always observed but realizations of $y_{i}$ are observed only when $s_{i}=1$. $^{2}$

Before proceeding we note that our general specification covers several models of special interest. Nonlinear models with additive unobservables, for example, can be represented as

$$
\mathcal{E}\left[y_{i} \mid x_{i}, u_{i}\right]=\mu\left(x_{i} ; \alpha_{0}\right)+u_{i} .
$$

Such models are used extensively, with the linear specification $\mu\left(x_{i} ; \alpha\right)=x_{i}^{\prime} \alpha$ being the leading case. Models with multiplicative unobservables are also covered. For non-negative limited dependent variables such models can be written as

$$
\mathcal{E}\left[y_{i} \mid x_{i}, u_{i}\right]=\varphi\left(x_{i} ; \beta_{0}\right) u_{i}
$$

where $u_{i} \geq 0$ and $\varphi$ maps to the positive real half-line. A prototypical specification for count data - such as the Poisson and the negative binomial models - would have $\varphi\left(x_{i} ; \beta\right)=$ $\exp \left(x_{i}^{\prime} \beta\right)$. A binary-choice model where $\varphi\left(x_{i} ; \beta\right)=G\left(x_{i}^{\prime} \beta\right)$ for some distribution function $G$ is equally covered here; see Wooldridge (1997, Example 4.2) for a motivation of such a specification.

Because sampling will not provide information on the distribution of $y_{i}$ given $s_{i}=0$, sample selection complicates inference on $\theta_{0}$. To see how the problem manifests itself, observe that

$$
\mathcal{E}^{*}\left[y_{i} \mid x_{i}, z_{i}\right]=\mu\left(x_{i} ; \alpha_{0}\right)+\varphi\left(x_{i} ; \beta_{0}\right) \mathcal{E}^{*}\left[u_{i} \mid x_{i}, z_{i}\right],
$$

where $\mathcal{E}^{*}$ refers to an expectation concerning the subpopulation for which $s_{i}=1$. Given the threshold-crossing structure of the selection rule and independence of $\left(u_{i}, v_{i}\right)$ from the observables covariates, we can further dissect the influence of the sample selection by using

$$
\lambda\left(p_{i}\right) \equiv \frac{\int_{-\infty}^{p_{i}} \int_{-\infty}^{+\infty} u f(u, v) \mathrm{d} u \mathrm{~d} v}{F_{v}\left(p_{i}\right)}=\mathcal{E}^{*}\left[u_{i} \mid p_{i}\right]
$$

where $f$ denotes the joint density of $\left(u_{i}, v_{i}\right)$ and $F_{v}$ is the marginal distribution of $v_{i}$. Indeed,

$$
\mathcal{E}^{*}\left[y_{i} \mid x_{i}, p_{i}\right]=\mu\left(x_{i} ; \alpha_{0}\right)+\varphi\left(x_{i} ; \beta_{0}\right) \lambda\left(p_{i}\right) .
$$

If $u_{i}$ and $v_{i}$ are independent, $\lambda$ is constant. Otherwise, $\lambda$ depends on the data through the index driving the propensity score of selection. In either case, $\mathcal{E}\left[y_{i} \mid x_{i}\right] \neq \mathcal{E}^{*}\left[y_{i} \mid x_{i}\right]$, in general. This implies that an estimation strategy that does not account for sample selection will typically suffer from misspecification bias in the sense of Heckman (1979). Furthermore,

$$
\lambda\left(p_{i}\right)=-\rho \sigma_{u} \frac{\phi\left(-p_{i} / \sigma_{v}\right)}{1-\Phi\left(-p_{i} / \sigma_{v}\right)} \quad \text { if } \quad\left(\begin{array}{c}
u_{i} \\
v_{i}
\end{array}\right) \sim \mathcal{N}\left(\left(\begin{array}{c}
0 \\
0
\end{array}\right),\left(\begin{array}{cc}
\sigma_{u}^{2} & \rho \sigma_{u} \sigma_{v} \\
\rho \sigma_{u} \sigma_{v} & \sigma_{v}^{2}
\end{array}\right)\right)
$$

${ }^{2}$ The analysis can easily be extended to situations in which $x_{i}$, too, is only observed when $s_{i}=1$. 
which is the correction term originally derived by Heckman (1979) in the context of the linear model. However, (2.3)-(2.4) hold without restricting $f$ to belong to a known parametric family of density functions. They also stretch beyond the conventional linear specification, and so may be of use to construct semiparametric inference techniques for models with multiplicative errors.

Our approach is inspired by the work of Powell (1987) and Ahn and Powell (1993) on pairwise differencing and builds on moment conditions that are similar in spirit to the ones considered by Chamberlain (1992) and Wooldridge (1997) in a different context. As

$$
\mathcal{E}^{*}\left[\tau_{i}\left(\theta_{0}\right) \mid x_{i}, p_{i}\right]=\lambda\left(p_{i}\right), \quad \tau_{i}(\theta) \equiv \frac{y_{i}-\mu\left(x_{i} ; \alpha\right)}{\varphi\left(x_{i} ; \beta\right)}
$$

follows from re-arranging (2.4), we have that

$$
\mathcal{E}^{*}\left[\tau_{i}\left(\theta_{0}\right) \mid x_{i}, p_{i}\right]-\mathcal{E}^{*}\left[\tau_{j}\left(\theta_{0}\right) \mid x_{j}, p_{j}\right]=\lambda\left(p_{i}\right)-\lambda\left(p_{j}\right)
$$

for any pair $i, j$. If $\lambda$ is a smooth function, the right-hand side of this expression will converge to zero as $\left|p_{i}-p_{j}\right| \rightarrow 0$. This suggests an approach based on moment conditions defined on the product space of the random variables in question that difference-out the selection bias. For clarity we use $\mathscr{E}$ rather than $\mathcal{E}$ to indicate the expectations operator with respect to a product measure, and again use $\mathscr{E}^{*}$ as a shorthand to refer to expectations that relate to the selected subpopulation. Now, introduce the random variable $\Delta_{i j} \equiv p_{i}-p_{j}$ on the product space. If

$$
\left|\lambda\left(p_{i}\right)-\lambda\left(p_{j}\right)\right| \leq \Lambda\left(p_{i}, p_{j}\right)\left|\Delta_{i j}\right|
$$

for some function $\Lambda$, then

$$
\left|\mathscr{E}^{*}\left[\tau_{i}\left(\theta_{0}\right)-\tau_{j}\left(\theta_{0}\right) \mid x_{i}, x_{j}, \Delta_{i j}\right]\right| \leq \mathscr{E}^{*}\left[\Lambda\left(p_{i}, p_{j}\right) \mid x_{i}, x_{j}, \Delta_{i j}\right]\left|\Delta_{i j}\right| \stackrel{\left|\Delta_{i j}\right| \downarrow 0}{\longrightarrow} 0
$$

provided that $\mathscr{E}^{*}\left[\Lambda\left(p_{i}, p_{j}\right) \mid x_{i}, x_{j}, \Delta_{i j}\right]$ exists for $\Delta_{i j}$ in a neighborhood of zero. This is a fairly weak condition. For example, if $\lambda$ is everywhere differentiable with derivative $\lambda^{\prime}$ we can take

$$
\Lambda\left(p_{i}, p_{j}\right)=\sup _{p \in\left[p_{i}, p_{j}\right]}\left|\lambda^{\prime}(p)\right|
$$

and a tail condition on this quantity allows the use of a dominated-convergence argument to establish the existence of the expectation. If $\lambda^{\prime}$ is also continuous, then it is locally Lipschitz and, therefore, locally bounded. This would equally imply the required condition to hold for sufficiently small $\Delta_{i j}$. By Leibniz's rule,

$$
\lambda^{\prime}\left(p_{i}\right)=\left(\mathcal{E}\left[u_{i} \mid v_{i}=p_{i}\right]-\lambda\left(p_{i}\right)\right) r\left(p_{i}\right)
$$

where $r\left(p_{i}\right) \equiv f_{v}\left(p_{i}\right) / F_{v}\left(p_{i}\right)$ is the inverse Mills ratio of $v_{i}$, and so $\lambda^{\prime}$ will be locally bounded if $\mathcal{E}\left[u_{i} \mid v_{i}=p_{i}\right], \lambda\left(p_{i}\right)$, and $r\left(p_{i}\right)$ are continuous. Although routinely done, demanding $\lambda^{\prime}$ to 
be uniformly bounded is somewhat too strong a requirement, particularly if $p$ can take on values on the whole real line. For example, when $\left(u_{i}, v_{i}\right)$ are jointly normal,

$$
\lambda^{\prime}\left(p_{i}\right)=\left(\rho \frac{\sigma_{u}}{\sigma_{v}} p_{i}+\rho \sigma_{u} \frac{\phi\left(-p_{i} / \sigma_{v}\right) / \sigma_{v}}{1-\Phi\left(-p_{i} / \sigma_{v}\right)}\right) \frac{\phi\left(-p_{i} / \sigma_{v}\right) / \sigma_{v}}{1-\Phi\left(-p_{i} / \sigma_{v}\right)} .
$$

Here, the magnitude of all terms increases without bound when $p_{i} \rightarrow-\infty$ and, indeed, $\lambda\left(p_{i}\right)$ itself diverges in this case. A similar pattern arises in the more general case of logconcave symmetric densities, for which the inverse Mills ratio is known to be monotonically decreasing in $p_{i}$.

The above argument suggests a strategy based on unconditional moment conditions in which more weight is assigned to pairs of variables for which $\Delta_{i j}$ lies in a shrinking neighborhood of zero. More precisely, for some suitable transformation function $\omega$, let $\omega\left(x_{i}, x_{j}\right)$ denote instrumental variables. ${ }^{3}$ Many candidate functions exist that transform conditional-moment equalities into unconditional ones without any information loss (see, e.g., Stinchcombe and White 1998 and Domínguez and Lobato 2004). Further, let $\kappa$ be a symmetric kernel function and let $\varsigma$ be an associated bandwidth. Then, under conventional regularity conditions introduced below,

$$
\mathscr{E}^{*}\left[\frac{\omega\left(x_{i}, x_{j}\right)\left(\tau_{i}\left(\theta_{0}\right)-\tau_{j}\left(\theta_{0}\right)\right)}{\varsigma} \kappa\left(\frac{\Delta_{i j}}{\varsigma}\right)\right] \stackrel{|\varsigma| \downarrow 0}{\longrightarrow} 0 .
$$

Overidentification is allowed for in the sense that the number of moments, say $m$, can exceed $\operatorname{dim} \theta$. A sample counterpart of these moment conditions is readily constructed using any of a number of available first-stage estimators of the selection equation, and can be combined easily through a GMM procedure to yield a two-step semiparametric estimator of $\theta_{0}$. This estimator will be the topic of the next subsection.

Because our approach relies on pairwise differencing, the parameters associated with regressors that are constant across observations will not be identified from (2.5). The leading example would be an intercept in $\mu\left(x_{i} ; \alpha\right)$. This is not surprising. Even in the classical linear sample-selection model, recovering the intercept term in a semiparametric fashion requires an argument involving identification at infinity, and yields estimators with non-standard properties (Andrews and Schafgans 1998).

Deriving sufficient conditions for global identification is difficult in models that are specified by a set of nonlinear moment conditions (see, e.g., Hall 2005, Section 3.1, for a discussion). The general model entertained here is a case in point. However, because our analysis is based on moment conditions that are conditional on the difference between the $p_{i}$, it is intuitive that we will need sufficient variation in the $x_{i}$ for given values of $p_{i}$. For example, in the standard linear model, where $\mu\left(x_{i} ; \alpha\right)=x_{i}^{\prime} \alpha$ and we set $\omega\left(x_{i}, x_{j}\right)=x_{i}-x_{j}$, it can be verified using (2.8) below that, if

$$
\operatorname{rank} \mathcal{E}^{*}\left[\operatorname{var}^{*}\left(x_{i} \mid p_{i}\right) f^{*}\left(p_{i}\right)\right]=\operatorname{dim} \alpha,
$$

\footnotetext{
${ }^{3}$ The analysis to follow can be extended to allow $\omega$ to depend on $\theta$. We could equally extend the definition of $\omega$ by allowing it to depend on variables other than the covariates $x_{i}$, which we do not do here for notational simplicity.
} 
then $\alpha_{0}$ is globally identified. Here, $f^{*}$ denotes the density of $p_{i}$ given selection, and $\operatorname{var}^{*}\left(x_{i} \mid p_{i}\right)$ is the conditional variance of $x_{i}$ given $p_{i}$ in the selected subpopulation. Although this condition can be satisfied because of nonlinearity, the key message to take away from it is that credible identification requires the presence of instrumental variables in the selection equation.

Local identification is easier to study. For example, in the general nonlinear model with additive unobservables, again with $\omega\left(x_{i}, x_{j}\right)=x_{i}-x_{j}$, local identification is achieved when

$$
\operatorname{rank} \mathcal{E}^{*}\left[\operatorname{cov}^{*}\left(x_{i}, \mu^{\prime}\left(x_{i} ; \alpha_{0}\right) \mid p_{i}\right) f^{*}\left(p_{i}\right)\right]=\operatorname{dim} \alpha,
$$

where $\mu^{\prime}$ is the first-derivative vector of $\mu$ with respect to $\alpha$. Of course, in the linear case, this boils down to the rank condition given earlier. As an example of a model with multiplicative errors, consider the exponential regression model from above. In this case, use of (2.8) shows that, if

$$
\operatorname{rank} \mathcal{E}^{*}\left[\lambda\left(p_{i}\right) \operatorname{var}^{*}\left(x_{i} \mid p_{i}\right) f^{*}\left(p_{i}\right)\right]=\operatorname{dim} \beta,
$$

then $\beta_{0}$ is locally identified. Furthermore, because local identification is equivalent to the Jacobian of the population moments having full rank at $\theta_{0}$, it can be empirically tested by applying any of a battery of rank tests to a plug-in version of this matrix. For example, Kleibergen and Paap (2006) provide a simple test statistic that will be applicable to our setup.

\subsection{Estimation}

For conciseness I will work with a linear-index specification for the propensity score, that is, I set $p_{i} \equiv p\left(z_{i}\right)=z_{i}^{\prime} \gamma_{0}$ for an unknown finite-dimensional parameter value $\gamma_{0}$. Flexible specifications of this form that include power transforms and interaction terms between regressors are common practice in empirical work. The distribution theory below could be extended to allow for a nonparametric selection rule at the cost of stronger smoothness requirements and more cumbersome notation.

Without loss of generality, take $\omega\left(x_{i}, x_{j}\right)$ to be antisymmetric in its arguments. Then a feasible empirical counterpart to the moment condition in (2.5) is

$$
\widehat{q}_{n}(\theta) \equiv\left(\begin{array}{l}
n \\
2
\end{array}\right)^{-1} \sum_{i=1}^{n} \sum_{i<j} \frac{\omega\left(x_{i}, x_{j}\right)\left(\tau_{i}(\theta)-\tau_{j}(\theta)\right)}{\varsigma} \kappa\left(\frac{\widehat{p}_{i}-\widehat{p}_{j}}{\varsigma}\right) s_{i} s_{j}
$$

where $\widehat{p}_{i} \equiv z_{i}^{\prime} \gamma_{n}$ and $\gamma_{n}$ is a consistent estimator of $\gamma_{0}$. Our GMM estimator of $\theta_{0}$ is the minimizer of a quadratic form in $\widehat{q}_{n}(\theta)$. It is given by

$$
\theta_{n} \equiv \arg \min _{\theta \in \Theta} \widehat{q}_{n}(\theta)^{\prime} V_{n} \widehat{q}_{n}(\theta)
$$

for a chosen symmetric positive-definite matrix $V_{n}$ of conformable dimension that serves to weight the moment conditions when $m>\operatorname{dim} \theta$, and a suitably defined parameter space 
$\Theta$ over which the minimization is performed. The interpretation of the kernel weight is immediate; pairs of observations for which $\left|\widehat{p}_{i}-\widehat{p}_{j}\right|$ is smaller receive a higher weight. Letting $\varsigma$ decrease with $n$ ensures that, asymptotically, only observations for which this difference converges to zero are taken into account.

One attractive feature of estimation based on pairwise differencing is that the function $\lambda$ need not be estimated. Alternative approaches to estimating $\theta_{0}$ could be devised that replace $\lambda$ in (2.4) by a nonparametric kernel or series estimator, and subsequently estimate $\theta_{0}$ via a semiparametric least-squares procedure. Such an approach would be in line with the work of Robinson (1988), Lee (2007), and Newey (2009). Contrary to the approach taken here, however, it does not generalize easily to the panel-data context. Furthermore, if an estimator of $\lambda(p)$ is desired, we may use

$$
\lambda_{n}(p) \equiv \frac{\sum_{i=1}^{n} \tau_{i}\left(\theta_{n}\right) \kappa\left(\frac{\widehat{p}_{i}-p}{\varsigma}\right) s_{i}}{\sum_{i=1}^{n} \kappa\left(\frac{\widehat{p}_{i}-p}{\varsigma}\right) s_{i}},
$$

for example. Of course, a series estimator would be equally well suited for this purpose. Under the conditions spelled out below, $\theta_{n}$ will be $\sqrt{n}$-consistent. From this it follows that the asymptotic behavior of $\lambda_{n}$ is not affected by the estimation noise in $\theta_{n}$, so inference on $\lambda$ using $\lambda_{n}$ can be performed using standard tools from nonparametric conditional-mean estimation. The empirical distribution function of the $\lambda_{n}\left(\widehat{p}_{i}\right)$ may be of interest. For example, if $\lambda_{n}\left(\widehat{p}_{i}\right)$ is found to vary substantially across $i$, this provides evidence on the presence of sample selection.

We now state the conditions under which we will derive distribution theory for $\theta_{n}$. The first condition imposes standard regularity conditions.

ASSUMPTION 2.1 (REgUlarity). The space $\Theta$ is compact and $\theta_{0}$ lies in its interior. Equation (2.5) identifies $\theta_{0}$, and $\mu$ and $\varphi$ are twice continuously differentiable in $\theta$. The $p_{i}$ are absolutely continuous.

The second assumption concerns the first-stage estimator .

Assumption 2.2 (FIRST STEP). The first-stage estimator, $\gamma_{n}$, is $\sqrt{n}$-consistent, and

$$
\sqrt{n}\left(\gamma_{n}-\gamma_{0}\right)=\frac{1}{\sqrt{n}} \sum_{i=1}^{n} \psi_{i}+o_{p}(1)
$$

for independent and identically distributed random variables $\psi_{i}$ that have zero mean and finite fourth-order moment.

Assumption 2.2 states that $\gamma_{n}$ must satisfy an asymptotic-linearity condition. This is not very demanding, as most semiparametric candidates for $\gamma_{n}$ do so; Powell (1994) provides a long list of eligible approaches.

Our third assumption collects standard restrictions on the kernel function and postulates eligible bandwidth choices. 
ASSUMPTION 2.3 (KERNEL). The kernel function $\kappa$ is bounded and twice continuously differentiable with bounded derivatives $\kappa^{\prime}$ and $\kappa^{\prime \prime}$, symmetric, and integrates to one. For some integer $k, \int_{-\infty}^{+\infty}\left|\varepsilon^{h}\right||\kappa(\varepsilon)| \mathrm{d} \varepsilon=0$ for all $0<h<k, \int_{-\infty}^{+\infty}|\kappa(\varepsilon)| \mathrm{d} \varepsilon<+\infty$, and $\int_{-\infty}^{+\infty}\left|\varepsilon^{k}\right||\kappa(\varepsilon)| \mathrm{d} \varepsilon<+\infty$. The bandwidth satisfies $\varsigma \propto n^{-r}$ for $\frac{1}{2 k}<r<\frac{1}{6}$.

Assumption 2.3 requires $\kappa$ to be a higher-order kernel. An eligible bandwidth sequence can be constructed as soon as $k>3$. Higher-order kernels are used to ensure that the limit distribution of $\theta_{n}$ is free of asymptotic bias. They are easy to construct, especially given that $\kappa$ is taken to be symmetric (see Li and Racine 2007, Section 1.11). Müller (1984) provides formulae to do so. For example, a fourth-order kernel based on the standard-normal density is

$$
\kappa(\varepsilon)=\left(\frac{3}{2}-\frac{1}{2} \varepsilon^{2}\right) \phi(\varepsilon),
$$

and is easily shown to satisfy the conditions in Assumption 2.3. We note that the use of a higher-order kernel is not required for consistency, and that $r<\frac{1}{4}$ suffices for such a purpose.

The fourth assumption contains moment restrictions that are needed to ensure uniform convergence of the objective function to its large-sample counterpart, and are thus required for consistency. Let

$$
\zeta_{i}\left(p_{j} ; \theta\right)=\left(\mathcal{E}^{*}\left[\omega\left(x_{i}, x_{j}\right) \mid p_{j}\right] \tau_{i}(\theta)+A_{i}\left(p_{j} ; \theta\right)-B_{i}\left(p_{j} ; \theta\right) \lambda\left(p_{j}\right)\right) d_{i}\left(p_{j}\right),
$$

where $d_{i}\left(p_{j}\right) \equiv s_{i} f^{*}\left(p_{j}\right) \operatorname{Pr}\left[s_{i}=1\right]$ and

$$
A_{i}\left(p_{j} ; \theta\right) \equiv \mathcal{E}^{*}\left[\omega\left(x_{i}, x_{j}\right) \frac{\mu\left(x_{j} ; \alpha\right)-\mu\left(x_{j} ; \alpha_{0}\right)}{\varphi\left(x_{j} ; \beta\right)} \mid p_{j}\right], B_{i}\left(p_{j} ; \theta\right) \equiv \mathcal{E}^{*}\left[\omega\left(x_{i}, x_{j}\right) \frac{\varphi\left(x_{j} ; \beta_{0}\right)}{\varphi\left(x_{j} ; \beta\right)} \mid p_{j}\right] .
$$

Also, let $\tau^{\prime}$ denote the first derivative of $\tau$ with respect to $\theta$. Denote the Euclidean norm and the Frobenius norm by $\|\cdot\|$.

Assumption 2.4 (Finite moments). For each $\theta \in \Theta, \mathcal{E}^{*}\left[\tau_{i}(\theta)^{8}\right]$ and $\mathcal{E}^{*}\left[\left\|\tau_{i}^{\prime}(\theta)\right\|^{4}\right]$ are finite. Both $\mathscr{E}\left[\left\|\omega\left(x_{i}, x_{j}\right)\right\|^{8}\right]$ and $\mathcal{E}\left[\left\|z_{i}\right\|^{4}\right]$ are finite. The function $\zeta_{i}(p ; \theta)$ is continuous in $p$ and $\mathcal{E}\left[\sup _{p}\left\|\zeta_{i}(p ; \theta)\right\|\right]$ is finite for each $\theta \in \Theta$.

The moment conditions ensure the variance of the empirical moment and its derivatives with respect to $\gamma$ and $\theta$ to exist. The dominance condition on $\left\|\zeta_{i}(p ; \theta)\right\|$ is standard in nonparametric estimation. Note from the form of $\zeta_{i}(p ; \theta)$ that it can be interpreted as a restriction on its tail behavior. This condition is needed for convergence of the kernelweighted objective function as $n$ diverges; see, e.g., Hansen (2008) for the application of such conditions in generic problems.

The fifth assumption is used to derive the limit distribution of $\sqrt{n}\left(\theta_{n}-\theta_{0}\right)$. Introduce

$$
\zeta_{i}^{\prime}(p ; \theta) \equiv \frac{\partial \zeta_{i}(p ; \theta)}{\partial \theta^{\prime}}, \quad \nabla_{h} \zeta_{i}(p ; \theta) \equiv \frac{\partial^{h} \zeta_{i}(p ; \theta)}{\partial p^{h}},
$$


Assumption 2.5 (smoothness). For each $\theta \in \Theta, \mathcal{E}^{*}\left[\left\|\tau_{i}^{\prime \prime}(\theta)\right\|^{4}\right]$ is finite. For each $\theta \in \Theta$, the function $\zeta_{i}^{\prime}(p ; \theta)$ is continuous in $p$ and $\mathcal{E}\left[\sup _{p}\left\|\zeta_{i}^{\prime}(p ; \theta)\right\|\right]$ is finite. $\nabla_{h} \zeta_{i}\left(p ; \theta_{0}\right)$ exists and $\mathcal{E}\left[\sup _{p}\left\|\nabla_{h} \zeta_{i}\left(p ; \theta_{0}\right)\right\|^{2}\right]$ are finite for all integers $h \leq k+1$.

The conditions on $\tau^{\prime \prime}$ and $\zeta_{i}^{\prime}(p ; \theta)$ are needed to establish convergence of the Jacobian of the moment conditions uniformly on $\Theta$. The higher-order smoothness requirements are needed to ensure the limit distribution of $\sqrt{n}\left(\theta_{n}-\theta_{0}\right)$ to be free of asymptotic bias. Such an approach to bias control is common in inference problems of this type. To interpret these restrictions, observe that

$$
\zeta_{i}\left(p_{j} ; \theta_{0}\right)=\mathcal{E}^{*}\left[\omega\left(x_{i}, x_{j}\right) \mid p_{j}\right]\left[\tau_{i}\left(\theta_{0}\right)-\lambda\left(p_{j}\right)\right] d_{i}\left(p_{j}\right)
$$

Assumption 2.5 then requires that $\mathcal{E}^{*}\left[\omega\left(x_{i}, x_{j}\right) \mid p_{j}\right], f^{*}\left(p_{j}\right)$, and $\lambda\left(p_{j}\right)$ are at least five times differentiable and also restricts the tail behavior of these quantities and their respective derivatives.

To state the limit distribution of the estimator, let $Q_{0} \equiv \mathcal{E}\left[\zeta_{i}^{\prime}\left(p_{i} ; \theta_{0}\right)\right]$ and introduce

$$
\sigma_{i} \equiv \zeta_{i}\left(p_{i} ; \theta_{0}\right)+H_{0} \psi_{i}
$$

for $H_{0} \equiv-\mathcal{E}\left[\nabla_{1} \zeta_{j}\left(p_{j} ; \theta_{0}\right) z_{j}^{\prime}\right]$; note that $\mathcal{E}\left[\sigma_{i}\right]=0$ and that $\Sigma \equiv \mathcal{E}\left[\sigma_{i} \sigma_{i}^{\prime}\right]<+\infty$.

Theorem 2.1 (Asymptotic Distribution). Let Assumptions 2.1-2.5 hold. Suppose that $\Sigma$ is positive definite, that $Q_{0}$ has full column rank, and that $V_{n} \stackrel{P}{\rightarrow} V_{0}$ for $V_{0}$ positive definite. Then $\sqrt{n}\left\|\theta_{n}-\theta_{0}\right\|=O_{P}(1)$ and

$$
\sqrt{n}\left(\theta_{n}-\theta_{0}\right) \stackrel{L}{\rightarrow} \mathcal{N}(0, \Upsilon), \quad \Upsilon \equiv 4\left(Q_{0}^{\prime} V_{0} Q_{0}\right)^{-1}\left(Q_{0}^{\prime} V_{0} \Sigma V_{0} Q_{0}\right)\left(Q_{0}^{\prime} V_{0} Q_{0}\right)^{-1}
$$

In particular, if $V_{n} \stackrel{P}{\rightarrow} \Sigma^{-1}$, then $\Upsilon=4\left(Q_{0}^{\prime} \Sigma^{-1} Q_{0}\right)^{-1}$.

A choice for $V_{n}$ so that $V_{n} \stackrel{P}{\rightarrow} \Sigma^{-1}$ is well known to be optimal in terms of asymptotic efficiency for a given set of moment conditions (Sargan 1958; Hansen 1982).

An estimator of the asymptotic variance matrix in Theorem 2.1 is needed to perform inference. An estimator of $V_{0}$ is available from the outset in the form of $V_{n}$. Estimators of $Q_{0}$ and $\Sigma$ can be constructed via the plug-in principle. Moreover,

$$
\begin{aligned}
Q_{n} & \equiv\left(\begin{array}{l}
n \\
2
\end{array}\right)^{-1} \sum_{i=1}^{n} \sum_{i<j} \frac{\omega\left(x_{i}, x_{j}\right)\left(\tau_{i}^{\prime}\left(\theta_{n}\right)-\tau_{j}^{\prime}\left(\theta_{n}\right)\right)^{\prime}}{\varsigma} \kappa\left(\frac{\widehat{p}_{i}-\widehat{p}_{j}}{\varsigma}\right) s_{i} s_{j}, \\
H_{n} & \equiv\left(\begin{array}{l}
n \\
2
\end{array}\right)^{-1} \sum_{i=1}^{n} \sum_{i<j} \frac{\omega\left(x_{i}, x_{j}\right)\left(\tau_{i}\left(\theta_{n}\right)-\tau_{j}\left(\theta_{n}\right)\right)}{\varsigma} \kappa^{\prime}\left(\frac{\widehat{p}_{i}-\widehat{p}_{j}}{\varsigma}\right) \frac{\left(z_{i}-z_{j}\right)^{\prime}}{\varsigma} s_{i} s_{j},
\end{aligned}
$$

constitute consistent estimators of the matrices $Q_{0}$ and $H_{0}$, respectively. An estimator of $\Sigma$ then is $\Sigma_{n} \equiv n^{-1} \sum_{i=1}^{n} \widehat{\sigma}_{i} \widehat{\sigma}_{i}^{\prime}$ for

$$
\widehat{\sigma}_{i} \equiv \widehat{\zeta}_{i}+H_{n} \widehat{\psi}_{i}, \quad \widehat{\zeta}_{i} \equiv \frac{1}{n-1} \sum_{j \neq i} \frac{\omega\left(x_{i}, x_{j}\right)\left(\tau_{i}\left(\theta_{n}\right)-\tau_{j}\left(\theta_{n}\right)\right)}{\varsigma} \kappa\left(\frac{\widehat{p}_{i}-\widehat{p}_{j}}{\varsigma}\right) s_{i} s_{j},
$$


where $\widehat{\psi}_{i}$ is an estimator of the influence function of $\gamma_{n}$. The precise form of this estimator will depend on the first-stage estimator used.

The following theorem permits the construction of asymptotically-valid test procedure based on the Wald principle.

Theorem 2.2 (Inference). Let Assumptions 2.1-2.5 hold. Suppose that $\Sigma$ is positive definite and that $Q_{0}$ has full column rank. Then

$$
\Upsilon_{n} \stackrel{P}{\rightarrow} \Upsilon, \quad \Upsilon_{n} \equiv 4\left(Q_{n}^{\prime} V_{n} Q_{n}\right)^{-1}\left(Q_{n}^{\prime} V_{n} \Sigma_{n} V_{n} Q_{n}\right)\left(Q_{n}^{\prime} V_{n} Q_{n}\right)^{-1}
$$

if $V_{n} \stackrel{P}{\rightarrow} V_{0}$ for $V_{n}$ and $V_{0}$ positive definite, and we assume that $n^{-1} \sum_{i=1}^{n}\left\|\widehat{\psi}_{i}-\psi_{i}\right\|^{2}=o_{P}(1)$.

Other consequences of Theorem 2.2 are the feasibility of the two-step GMM estimator as well as the fact that

$$
n \widehat{q}_{n}\left(\theta_{n}\right)^{\prime} \Sigma_{n}^{-1} \widehat{q}_{n}\left(\theta_{n}\right) \stackrel{P}{\rightarrow} \chi_{m-\operatorname{dim} \theta}^{2}
$$

for the optimally-weighted estimator. This justifies the asymptotic validity of the usual overidentification tests (Hansen 1982). We also note that, under the conditions stated in Lemma 3.4 and Lemma 3.5 of Pakes and Pollard (1989), all results continue to hold for the continuously-updated version of $\theta_{n}$ (Hansen, Heaton, and Yaron 1996).

\subsection{Simulations}

We next discuss the results from a Monte Carlo experiment where $y_{i}$ given $\left(x_{i}, u_{i}\right)$ is a Poisson variate with mean

$$
\mathcal{E}\left[y_{i} \mid x_{i}, u_{i}\right]=\exp \left(c+x_{i} \beta_{0}\right) u_{i}
$$

where $c$ is a constant. Let $w_{i} \equiv \log u_{i}$. We generate

$$
\left(\begin{array}{l}
w_{i} \\
v_{i}
\end{array}\right) \sim \mathcal{N}\left(\left(\begin{array}{l}
0 \\
0
\end{array}\right),\left(\begin{array}{cc}
\sigma_{w}^{2} & \rho \sigma_{w} \\
\rho \sigma_{w} & 1
\end{array}\right)\right)
$$

for $|\rho|<1$, so that the marginal distribution of $u_{i}$ is log-normal and the selection equation is a conventional probit. The unconditional mean of $u_{i}$ equals $\exp \left(\sigma_{w}^{2} / 2\right)$, and so we set $c=-\sigma_{w}^{2} / 2$ to recenter its distribution at one. Then

$$
\mathcal{E}^{*}\left[y_{i} \mid x_{i}, p_{i}\right]=\exp \left(x_{i} \beta_{0}\right) \lambda\left(p_{i}\right), \quad \lambda\left(p_{i}\right)=\frac{\Phi\left(-\rho \sigma_{w}+p_{i}\right)}{\Phi\left(p_{i}\right)} ;
$$

see also Terza (1998). For reasons of parsimony, we set $p_{i}=x_{i}+\gamma_{0} a_{i}$ and $\operatorname{draw}\left(x_{i}, a_{i}\right)$ as

$$
\left(\begin{array}{l}
x_{i} \\
a_{i}
\end{array}\right) \sim \mathcal{N}\left(\left(\begin{array}{l}
0 \\
0
\end{array}\right),\left(\begin{array}{cc}
\sigma_{x}^{2} & \varrho \sigma_{x} \sigma_{a} \\
\varrho \sigma_{x} \sigma_{a} & 1
\end{array}\right)\right)
$$

for $|\varrho|<1$. This specification fixes the coefficient associated with $x_{i}$ to unity, which is a convenient normalization for our first-stage estimator. It also allows us to get a closed-form 
expression for $\mathcal{E}^{*}\left[\lambda\left(p_{i}\right) \mid x_{i}\right]$. Let

$$
\mu_{p} \equiv \frac{-\rho \sigma_{w}}{\sqrt{1+\left(\left|\gamma_{0}\right|(1-\varrho) \sigma_{a}\right)^{2}}}, \quad \sigma_{p} \equiv \frac{1+\gamma_{0} \varrho \sigma_{a} / \sigma_{x}}{\sqrt{1+\left(\left|\gamma_{0}\right|(1-\varrho) \sigma_{a}\right)^{2}}} .
$$

Then, after some algebra, we find that

$$
\mathcal{E}^{*}\left[y_{i} \mid x_{i}\right]=\exp \left(x_{i} \beta_{0}\right) \frac{\Phi\left(\mu_{p}+\sigma_{p} x_{i}\right)}{\Phi\left(\sigma_{p} x_{i}\right)}
$$

the calculation underlying this result equally reveals that $\operatorname{Pr}\left[s_{i}=1 \mid x_{i}\right]=\Phi\left(\sigma_{p} x_{i}\right)$. Thus, indeed, $\mathcal{E}^{*}\left[y_{i} \mid x_{i}\right] \neq \mathcal{E}\left[y_{i} \mid x_{i}\right]$ and an exponential-regression estimator will be inconsistent unless $\mu_{p}=0$, which holds only when either $\rho=0$ or $\sigma_{w}=0$. Below we consider inference on $\beta_{0}$ for different choices of the parameter values based on the vector of empirical moments

$$
\frac{1}{\varsigma}\left(\begin{array}{l}
n \\
2
\end{array}\right)^{-1} \sum_{i=1}^{n} \sum_{i<j}\left(\begin{array}{l}
x_{i}-x_{j} \\
a_{i}-a_{j}
\end{array}\right)\left(\frac{y_{i}}{\exp \left(x_{i} \beta\right)}-\frac{y_{j}}{\exp \left(x_{j} \beta\right)}\right) \kappa\left(\frac{\widehat{p}_{i}-\widehat{p}_{j}}{\varsigma}\right) s_{i} s_{j} .
$$

In this case $\beta_{0}$ is overidentified. We consider both one-step and two-step versions of our estimator; the one-step estimator minimizes the Euclidean norm of the empirical moments, the two-step estimator uses the one-step estimator to form a plug-in estimator of the optimal metric.

We use the maximum rank-correlation estimator of Han (1987) to construct estimates of the $p_{i}$. Although it is known not to reach the semiparametric efficiency bound for the binary-choice model, this estimator is a simple and robust choice that does not require the choice of tuning parameters. It is consistent and asymptotically-linear under weak conditions; see Sherman (1993). For the implementation of our weighting procedure we take $\kappa$ to equal the fourth-order kernel given in (2.7) and set $\varsigma=h_{n} n^{-1 / 7}$ for some scalar constant $h_{n}$; we will consider several choices for $h_{n}$. In any case, it is good practice to standardize the argument of the kernel function by its empirical standard deviation of $\widehat{p}_{i}$, and this is done throughout. To get an idea of the severity of the sample selection in each design we also consider one-step and two-step version of the naive GMM estimator that is based on an unweighted version of (2.9).

Simulation results for three designs are presented. The designs differ only in the severity of the sample-selection issue, by varying $\rho$. A full description of the designs is given below Tables 1 and 2. For each design we evaluate the performance of the estimators for $n=250$ and $n=500$. Note that $\operatorname{Pr}\left[s_{i}=1\right]=\frac{1}{2}$, so the effective sample sizes are only 125 and 250 , on average, which is fairly small for semiparametric techniques. We computed $\beta_{n}$ using four different but fixed values for $h_{n}$ to evaluate the sensitivity of the results to the bandwidth choice, and also using one automated choice. The latter bandwidth is obtained by minimizing

$$
\widehat{q}_{n}\left(\beta, h_{n}\right)^{\prime} V_{n} \widehat{q}_{n}\left(\beta, h_{n}\right)
$$

jointly with respect to $\left(\beta, h_{n}\right)$, using obvious notation. That is, we treat $h_{n}$ as a parameter to be estimated. This is similar to the proposal of Härdle, Hall, and Ichimura (1993) in 
Table 1. One-step estimators

\begin{tabular}{|c|c|c|c|c|c|c|c|c|c|c|c|c|c|}
\hline \multirow[t]{2}{*}{ 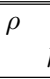 } & \multirow[t]{2}{*}{$\bar{n} n$} & \multicolumn{6}{|c|}{ bias } & \multicolumn{6}{|c|}{ standard deviation } \\
\hline & & $\sim$ & 1 & 1.5 & 2.5 & 3 & $\widehat{h}_{n}$ & $\sim$ & 1 & 1.5 & 2.5 & 3 & $\widehat{h}_{n}$ \\
\hline 0 & 250 & .017 & .020 & .019 & .018 & .018 & .018 & .188 & .299 & .288 & .236 & .214 & .271 \\
\hline 0 & 500 & .009 & .016 & .016 & .015 & .015 & .004 & .127 & .205 & .201 & .175 & .159 & .173 \\
\hline-.5 & 250 & -.202 & -.010 & -.016 & -.057 & -.084 & .000 & .167 & .274 & .264 & .217 & .195 & .255 \\
\hline-.5 & 500 & -.199 & .007 & .003 & -.033 & -.060 & .002 & .117 & .193 & .189 & .163 & .147 & .169 \\
\hline .5 & 250 & .281 & .038 & .047 & .105 & .139 & .030 & .205 & .307 & .296 & .247 & .230 & .232 \\
\hline .5 & 500 & .262 & .036 & .040 & .080 & .111 & .010 & .141 & .212 & .208 & .179 & .164 & .130 \\
\hline$\rho$ & $n$ & \multicolumn{6}{|c|}{ standard error to standard deviation } & \multicolumn{6}{|c|}{ rejection frequency } \\
\hline \multicolumn{2}{|c|}{$h_{n}$} & $\sim$ & 1 & 1.5 & 2.5 & 3 & $\widehat{h}_{n}$ & $\sim$ & 1 & 1.5 & 2.5 & 3 & $\widehat{h}_{n}$ \\
\hline 0 & 250 & .908 & 1.072 & 1.092 & 1.056 & 1.013 & 1.211 & .067 & .033 & .020 & .033 & .052 & .033 \\
\hline 0 & 500 & .948 & 1.071 & 1.065 & 1.065 & 1.036 & 1.260 & .065 & .030 & .022 & .019 & .031 & .038 \\
\hline-.5 & 250 & .911 & 1.122 & 1.157 & 1.132 & 1.046 & 1.217 & .320 & .037 & .028 & .060 & .085 & .031 \\
\hline-.5 & 500 & .931 & 1.079 & 1.079 & 1.121 & 1.055 & 1.228 & .453 & .044 & .036 & .046 & .067 & .034 \\
\hline .5 & 250 & .936 & 1.069 & 1.132 & 1.061 & .993 & 1.428 & .295 & .032 & .019 & .046 & .075 & .021 \\
\hline .5 & 500 & .961 & 1.042 & 1.061 & 1.102 & 1.036 & 1.690 & .486 & .038 & .036 & .053 & .081 & .032 \\
\hline
\end{tabular}

the semiparametric least-squares context, where it is known to possess certain optimality properties. Although we make no claim that such optimality carries over to the current setting we will find that this approach works quite well in our simulations. Tables 1 and 2 contain the bias, the standard deviation, the ratio of the average estimated standard error to the standard deviation, and the empirical rejection rate of $95 \%$-confidence intervals for $\beta_{0}$ for the one-step and the two-step GMM estimators, respectively, obtained over 1,000 Monte Carlo replications. The columns with $h_{n}=\sim$ refer to the naive estimator. The columns with $h_{n}=\widehat{h}_{n}$ relate to the estimator constructed by estimating the bandwidth as just described.

Both tables show that the naive estimator does well when sample selection is exogenous but suffers from large bias when it is not. Our estimator has much smaller bias for all designs considered. Like in standard nonparametric regression, the choice of $h_{n}$ affects both the bias and variance of the estimator. The larger $h_{n}$, the smaller the variance but the larger the bias, and vice versa. Nonetheless, the plug-in estimator of the asymptotic variance does quite well in capturing the variability of the estimator across the Monte Carlo replications for all choices of $h_{n}$. Similarly, the empirical rejection frquencies are close to their nominal value of .05 for all choices while the bias in the naive estimator implies poor coverage of the confidence intervals constructed from it.

\section{A semiparametric approach for panel data}

\subsection{The model and moment conditions}

Now consider a semiparametric model for group-level data with stratum-specific nuisance parameters. For independent groups $i=1,2 \ldots, n$, let $y_{i} \equiv\left(y_{i 1}, y_{i 2}\right)$ be outcomes whose conditional mean given observables $x_{i} \equiv\left(x_{i 1}, x_{i 2}\right)$, unobservables $u_{i} \equiv\left(u_{i 1}, u_{i 2}\right)$, and a 
Table 2. Two-step estimators

\begin{tabular}{|c|c|c|c|c|c|c|c|c|c|c|c|c|c|}
\hline \multirow[t]{2}{*}{$\overline{\rho \rho}$} & \multirow[t]{2}{*}{ 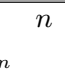 } & \multicolumn{6}{|c|}{ bias } & \multicolumn{6}{|c|}{ standard deviation } \\
\hline & & $\sim$ & 1 & 1.5 & 2.5 & 3 & $\widehat{h}_{n}$ & $\sim$ & 1 & 1.5 & 2.5 & 3 & $\widehat{h}_{n}$ \\
\hline 0 & 250 & .026 & .021 & .019 & .027 & .026 & .026 & .185 & .301 & .303 & .212 & .193 & .307 \\
\hline 0 & 500 & .013 & .016 & .015 & .016 & .015 & .006 & .124 & .203 & .204 & .152 & .131 & .207 \\
\hline-.5 & 250 & -.163 & -.009 & -.004 & -.109 & -.125 & .001 & .166 & .275 & .284 & .209 & .176 & .275 \\
\hline-.5 & 500 & -.161 & .006 & .010 & -.085 & -.134 & -.002 & .115 & .192 & .194 & .171 & .122 & .190 \\
\hline .5 & 250 & .271 & .039 & .032 & .196 & .191 & .044 & .204 & .312 & .317 & .233 & .211 & .315 \\
\hline .5 & 500 & .245 & .037 & .032 & .188 & .209 & .017 & .140 & .212 & .214 & .182 & .145 & .207 \\
\hline$\rho$ & $n$ & \multicolumn{6}{|c|}{ standard error to standard deviation } & \multicolumn{6}{|c|}{ rejection frequency } \\
\hline \multicolumn{2}{|c|}{$h_{n}$} & $\sim$ & 1 & 1.5 & 2.5 & 3 & $\widehat{h}_{n}$ & $\sim$ & 1 & 1.5 & 2.5 & 3 & $\widehat{h}_{n}$ \\
\hline 0 & 250 & .889 & .990 & .940 & 1.014 & .997 & .997 & .081 & .050 & .050 & .050 & .057 & .056 \\
\hline 0 & 500 & .935 & 1.041 & .993 & 1.029 & 1.056 & 1.013 & .074 & .036 & .038 & .028 & .042 & .045 \\
\hline-.5 & 250 & .886 & 1.025 & .966 & .944 & .996 & 1.026 & .277 & .055 & .062 & .124 & .140 & .046 \\
\hline-.5 & 500 & .913 & 1.035 & 1.000 & .872 & 1.011 & 1.040 & .375 & .049 & .059 & .152 & .217 & .043 \\
\hline .5 & 250 & .905 & .974 & .924 & 1.012 & 1.012 & .987 & .297 & .044 & .058 & .099 & .116 & .045 \\
\hline .5 & 500 & .929 & 1.002 & .970 & .950 & 1.055 & 1.023 & .457 & .048 & .055 & .184 & .230 & .047 \\
\hline
\end{tabular}

group-specific fixed effect $\eta_{i}$ is given by

$$
\mathcal{E}\left[y_{i j} \mid x_{i}, u_{i}, \eta_{i}\right]=\mu\left(x_{i j} ; \alpha_{0}\right)+\eta_{i} \varphi\left(x_{i j} ; \beta_{0}\right) u_{i j}
$$

The focus on two datapoints per group will simplify the subsequent exposition in terms of notational burden but is without loss of generality. A panel analog of the cross-sectional selection rule from above has

$$
\operatorname{Pr}\left[s_{i j}=1 \mid p_{i}, \iota_{i}\right]=\mathcal{E}\left[1\left\{p_{i j}+\iota_{i} \geq v_{i j}\right\} \mid p_{i}, \iota_{i}\right],
$$

where $p_{i} \equiv\left(p_{i 1}, p_{i 2}\right)$ with $p_{i j} \equiv z_{i j}^{\prime} \gamma_{0}$ for regressors $z_{i} \equiv\left(z_{i 1}, z_{i 2}\right)^{\prime}$, and $\iota_{i}$ a fixed effect. Interest again lies in consistently estimating the finite-dimensional parameter $\theta_{0}=\left(\alpha_{0}^{\prime}, \beta_{0}^{\prime}\right)^{\prime}$ under asymptotics where the number of groups, $n$, diverges.

Similar to before, let $\tau_{i j}(\theta) \equiv\left(y_{i j}-\mu\left(x_{i j} ; \alpha\right)\right) / \varphi\left(x_{i j} ; \beta\right)$. Suppose that $\left(u_{i}, v_{i}\right)$ are jointly independent of $\left(x_{i}, z_{i}\right)$ conditional on $\left(\eta_{i}, \iota_{i}\right)$. Then, if the $\left\{\left(u_{i 1}, v_{i 1}\right),\left(u_{i 2}, v_{i 2}\right)\right\}$ are exchangeable conditional on $\left(\eta_{i}, \iota_{i}\right)$,

$$
\mathcal{E}^{*}\left[\tau_{i 1}\left(\theta_{0}\right)-\tau_{i 2}\left(\theta_{0}\right) \mid x_{i}, p_{i}, \eta_{i}, \iota_{i}\right]=\eta_{i} \lambda_{i}\left(p_{i 1}+\iota_{i}, p_{i 2}+\iota_{i}\right)-\eta_{i} \lambda_{i}\left(p_{i 2}+\iota_{i}, p_{i 1}+\iota_{i}\right),
$$

where, now, the superscript on the expectations operator is a shorthand for the conditioning event $\left\{s_{i 1}=1, s_{i 2}=1\right\}$ holding, and

$$
\begin{aligned}
\lambda_{i}\left(p_{i 1}+\iota_{i}, p_{i 2}+\iota_{i}\right) & \equiv \mathcal{E}^{*}\left[u_{i 1} \mid v_{i 1} \leq p_{i 1}+\iota_{i}, v_{i 2} \leq p_{i 2}+\iota_{i}, \eta_{i}, \iota_{i}\right] \\
\lambda_{i}\left(p_{i 2}+\iota_{i}, p_{i 1}+\iota_{i}\right) & \equiv \mathcal{E}^{*}\left[u_{i 2} \mid v_{i 1} \leq p_{i 1}+\iota_{i}, v_{i 2} \leq p_{i 2}+\iota_{i}, \eta_{i}, \iota_{i}\right]
\end{aligned}
$$

The $i$ subscript on $\lambda$ stresses that the function can be heterogenous because the distribution of $\left(u_{i}, v_{i}\right)$ can vary with $i$ beyond its dependence on $\left(\eta_{i}, \iota_{i}\right)$. For example, if $\left(u_{i j}, v_{i j}\right)$ would be independent of $\left(\eta_{i}, \iota_{i}\right)$ and would be i.i.d. within groups, then $\lambda_{i}$ would simplify to

$$
\lambda_{i}\left(p_{i j}+\iota_{i}\right)=\frac{\int_{-\infty}^{p_{i j}+\iota_{i}} \int_{-\infty}^{+\infty} u f_{i}(u, v) \mathrm{d} u \mathrm{~d} v}{F_{i}\left(p_{i j}+\eta_{i}\right)}
$$


for $f_{i}$ the joint distribution of $\left(u_{i j}, v_{i j}\right)$ and $F_{i}$ the marginal distribution of the $v_{i j}$. This function varies with $i$ because $\iota_{i}$ and $f_{i}$ do.

In (3.3), the differencing is done within groups, not across groups. Indeed, the additional heterogeneity across $i$ that is allowed for here, as compared to the cross-sectional model, invalidates any approach based on the pairwise comparison of observations along the crosssectional dimension of the panel. However, exchangeability implies that, for $\Delta_{i} \equiv p_{i 1}-p_{i 2}$,

$$
\left|\lambda_{i}\left(p_{i 1}+\iota_{i}, p_{i 2}+\iota_{i}\right)-\lambda_{i}\left(p_{i 2}+\iota_{i}, p_{i 1}+\iota_{i}\right)\right| \stackrel{\left|\Delta_{i}\right| \downarrow 0}{\longrightarrow} 0
$$

provided $\lambda_{i}$ is a smooth function in the same sense as discussed above. This motivates a strategy that aims to recover $\theta_{0}$ from moment conditions of the form

$$
\mathcal{E}^{*}\left[\frac{\omega\left(x_{i 1}, x_{i 2}\right)\left(\tau_{i 1}\left(\theta_{0}\right)-\tau_{i 2}\left(\theta_{0}\right)\right)}{\varsigma} \kappa\left(\frac{\Delta_{i}}{\varsigma}\right)\right] \stackrel{|\varsigma| \downarrow 0}{\longrightarrow} 0,
$$

where we recycle notation for the kernel function $\kappa$ and bandwidth $\varsigma$, and retain $\omega\left(x_{i 1}, x_{i 2}\right)$ to indicate a vector of instrumental variables. We will provide distribution theory for estimators based on (3.4) in the next subsection.

Like in the cross-sectional case, these moment conditions can be linked to an approach to sample selection problem in the linear model, in this case Kyriazidou (1997, 2001); see also the work of Honoré and Kyriazidou (2000) for a related approach to a different problem. Indeed, Kyriazidou $(1997,2001)$ can be interpreted as a fixed-effect version of Powell (1987). It is, perhaps, useful to stress that the presence of fixed effects makes it difficult to extend the approach of Robinson (1988) and Newey (2009) to models for panel data. Indeed, an operational version of (3.4) requires only a consistent estimator of $\gamma_{0}$, but not of the $\iota_{i}$ nor the $\lambda_{i}$. The latter two cannot be constructed under asymptotics where the number of observations per group is treated as fixed. ${ }^{4}$

\subsection{Estimation}

Similar to before, (3.4) suggests estimating $\theta_{0}$ by a GMM estimator of the form

$$
\theta_{n}=\arg \min _{\theta \in \Theta} \widehat{q}_{n}(\theta)^{\prime} V_{n} \widehat{q}_{n}(\theta),
$$

where $V_{n}$ is a weight matrix and, now,

$$
\widehat{q}_{n}(\theta) \equiv \frac{1}{n} \sum_{i=1}^{n} \frac{\omega\left(x_{i 1}, x_{i 2}\right)\left(\tau_{i 1}(\theta)-\tau_{i 2}(\theta)\right)}{\varsigma} \kappa\left(\frac{\widehat{\Delta}_{i}}{\varsigma}\right) s_{i 1} s_{i 2}
$$

for $\widehat{\Delta}_{i} \equiv \widehat{p}_{i 1}-\widehat{p}_{i 2}, \widehat{p}_{i j} \equiv z_{i j}^{\prime} \gamma_{n}$, and $\gamma_{n}$ a first-stage estimator of $\gamma_{0}$. Although this estimator looks similar to the one introduced for the cross-sectional model above, its asymptotic

${ }^{4}$ Fernández-Val and Vella (2011) consider two-step estimation of a class of fixed-effect models under asymptotics where the number of groups and the number of observations per group diverge at the same rate. Implementation requires the distribution of $\left(u_{i j}, v_{i j}\right)$ to be parametrically specified. 
behavior is quite different. Most importantly, it will converge at the nonparametric rate $1 / \sqrt{n \varsigma}$ rather than at the parametric rate $1 / \sqrt{n}$. The reason for this is the need to resort to within-group differences rather than between-group differences. Indeed, here, $\widehat{q}_{n}(\theta)$ has the form of (the numerator of) a kernel-based nonparametric conditional-mean estimator, while it was a $U$-statistic of order two in the cross-sectional case. The smoothing that arises from the additional averaging is what leads to $1 / \sqrt{n}$ convergence in that case.

The conditions under which we will derive distribution theory for $\theta_{n}$ are provided next. The first assumption is again standard.

Assumption 3.1 (REgUlarity). The space $\Theta$ is compact and $\theta_{0}$ lies in its interior. Equation (3.4) identifies $\theta_{0}$, and $\mu$ and $\varphi$ are twice continuously differentiable in $\theta$. The $\Delta_{i}$ are absolutely continuous and its density given selection, $f^{*}$, is strictly positive in a neighborhood of zero.

Assumption 3.1 does not require stationarity of the data, but identification clearly requires that the support of $p_{i 1}$ and the support of $p_{i 2}$ overlap to some extent.

The next two assumptions deal with the first-stage estimator, and with the kernel and bandwidth, respectively.

Assumption 3.2 (FIRST Step). $\left\|\gamma_{n}-\gamma_{0}\right\|=O_{p}\left(n^{-s}\right)$ for $s \in(2 / 5,1 / 2]$.

ASSUMPTION 3.3 (KERNEL). The kernel function $\kappa$ is bounded and twice continuously differentiable with bounded derivatives $\kappa^{\prime}$ and $\kappa^{\prime \prime}$, symmetric, and integrates to one. For some integer $k, \int_{-\infty}^{+\infty}\left|\varepsilon^{h}\right||\kappa(\varepsilon)| \mathrm{d} \varepsilon=0$ for all $0<h<k, \int_{-\infty}^{+\infty}|\kappa(\varepsilon)| \mathrm{d} \varepsilon<+\infty$, and $\int_{-\infty}^{+\infty}\left|\varepsilon^{k}\right||\kappa(\varepsilon)| \mathrm{d} \varepsilon<+\infty$. Also, $\int_{-\infty}^{+\infty}|\kappa(\varepsilon)|^{2} \mathrm{~d} \varepsilon<+\infty$. The bandwidth satisfies $\varsigma \propto n^{-r}$ for $\max \left\{\frac{1}{1+2 k}, 1-2 s\right\}<r<\frac{s}{2}$.

Assumptions 3.2 and 3.3 are different from Assumptions 2.2 and 2.3. First, fixed-effect estimation of binary-choice models is known to be difficult, and possible at the parametric rate only in a logit specification (Chamberlain 2010). Thus, we need to allow for estimators that converge at a slower rate. Second, the permissible convergence rates of the bandwidth depend on the first-stage estimator. Under Assumptions 3.2 and 3.3, $\sqrt{n \varsigma}\left\|\gamma_{n}-\gamma_{0}\right\|=o_{P}(1)$, so $\theta_{n}$ will converge slower than $\gamma_{n}$, and the asymptotic variance of the former will not depend on the estimation noise in the latter. The rates in Assumption 3.2 allow for estimation by smoothed maximum score (Horowitz 1992; Charlier, Melenberg, and van Soest 1995) but rule out the original maximum-score estimator (Manski 1975, 1985, 1987), which converges at the rate $n^{-1 / 3}$.

To move on to the formulation of moment requirements and smoothness conditions, let

$$
\zeta\left(\Delta_{i} ; \theta\right) \equiv \mathcal{E}^{*}\left[\left\{A_{i 1}(\theta)-A_{i 2}(\theta)\right\}+\eta_{i}\left\{\lambda_{i}\left(p_{i 1}+\iota_{i}\right) B_{i 1}(\theta)-\lambda_{i}\left(p_{i 2}+\iota_{i}\right) B_{i 2}(\theta)\right\} \mid \Delta_{i}\right] d\left(\Delta_{i}\right),
$$

where, recalling that $f^{*}$ is the density of $\Delta_{i}$ given selection, $d\left(\Delta_{i}\right) \equiv f^{*}\left(\Delta_{i}\right) \operatorname{Pr}\left[s_{i 1} s_{i 2}=1\right]$, and

$$
A_{i j}(\theta) \equiv \omega\left(x_{i 1}, x_{i 2}\right) \frac{\mu\left(x_{i j} ; \alpha_{0}\right)-\mu\left(x_{i j} ; \alpha\right)}{\varphi\left(x_{i j} ; \beta\right)}, \quad B_{i j}(\theta) \equiv \omega\left(x_{i 1}, x_{i 2}\right) \frac{\varphi\left(x_{i j} ; \beta_{0}\right)}{\varphi\left(x_{i j} ; \beta\right)} .
$$


Consistency will follow from the following restrictions. The motivation for these conditions is as before.

Assumption 3.4 (Finite moments). For each $\theta \in \Theta$, both $\mathcal{E}^{*}\left[\left|\tau_{i 1}(\theta)-\tau_{i 2}(\theta)\right|^{6}\right]$ and $\mathcal{E}^{*}\left[\left\|\tau_{i 1}^{\prime}(\theta)-\tau_{i 2}^{\prime}(\theta)\right\|^{4}\right]$ are finite. Both $\mathcal{E}\left[\left\|\omega\left(x_{i 1}, x_{i 2}\right)\right\|^{6}\right]$ and $\mathcal{E}\left[\left\|z_{i 1}-z_{i 2}\right\|^{4}\right]$ are finite. The function $\zeta(\Delta ; \theta)$ is continuous in $\Delta$ in a neighborhood of zero and $\sup _{\Delta}\|\zeta(\Delta ; \theta)\|$ is finite for each $\theta \in \Theta$.

The moment conditions validate the use of laws of large numbers. The requirements on $\zeta\left(\Delta_{i} ; \theta\right)$ allow the use of a bounded-convergence argument to establish uniform consistency of the empirical moment. It can again be seen as a tail condition on the various conditional expectations involving $A_{i j}(\theta), B_{i j}(\theta), \eta_{i}$, and $\lambda_{i}$ that appear in it.

The next assumption is used to obtain asymptotic normality and zero asymptotic bias. To state it, again let

$$
\zeta^{\prime}(\Delta ; \theta) \equiv \frac{\partial \zeta(\Delta ; \theta)}{\partial \theta^{\prime}}, \quad \nabla_{h} \zeta(\Delta ; \theta) \equiv \frac{\partial^{h} \zeta(\Delta ; \theta)}{\partial \Delta^{h}},
$$

$Q_{0} \equiv \zeta^{\prime}\left(0 ; \theta_{0}\right)$, and $\tau^{\prime \prime}$ for the second-derivative matrix of $\tau$. The proof to asymptotic normality shows that, under Assumptions 3.1-3.5,

$$
\sqrt{n \varsigma} \widehat{q}_{n}\left(\theta_{0}\right) \stackrel{L}{\rightarrow} \mathcal{N}(0, \Sigma)
$$

where $\Sigma \equiv \Sigma\left(0 ; \theta_{0}\right) \int_{-\infty}^{+\infty}|\kappa(\varepsilon)|^{2} \mathrm{~d} \varepsilon$ for

$$
\Sigma\left(\Delta_{i} ; \theta_{0}\right) \equiv \mathcal{E}^{*}\left[\omega\left(x_{i 1}, x_{i 2}\right) \omega\left(x_{i 1}, x_{i 2}\right)^{\prime}\left(\tau_{i 1}\left(\theta_{0}\right)-\tau_{i 2}\left(\theta_{0}\right)\right)^{2} \mid \Delta_{i}\right] d\left(\Delta_{i}\right) .
$$

The expression for the asymptotic variance cannot be simplified further given that our model does not restrict the conditional variance of the $y_{i j}$ and does not rule out serial correlation in the $\left(u_{i j}, v_{i j}\right)$. Observe that, indeed, $\Sigma$ does not depend on the asymptotic variance of the first-stage estimator $\gamma_{n}$. We will need a technical restriction on the matrix

$$
H\left(\Delta_{i}\right) \equiv \mathcal{E}^{*}\left[\omega\left(x_{i 1}, x_{i 2}\right)\left(z_{i 1}-z_{i 2}\right)^{\prime} \eta_{i}\left\{\lambda_{i}\left(p_{i 1}+\iota_{i}\right)-\lambda_{i}\left(p_{i 2}+\iota_{i}\right)\right\} \mid \Delta_{i}\right] d\left(\Delta_{i}\right)
$$

to justify this formally. This matrix arises in an expansion of the empirical moment around $\gamma_{0}$.

Assumption 3.5 (smoothness). For each $\theta \in \Theta, \mathcal{E}^{*}\left[\left\|\tau_{i 1}^{\prime \prime}(\theta)-\tau_{i 2}^{\prime \prime}(\theta)\right\|^{4}\right]$ is finite and $\zeta^{\prime}(\Delta ; \theta)$ is continuous in $\Delta$, and $\sup _{\Delta}\left\|\zeta^{\prime}(\Delta ; \theta)\right\|$ is finite. $\Sigma\left(\Delta ; \theta_{0}\right)$ is continuous in $\Delta$ in a neighborhood of zero, $\sup _{\Delta}\left\|\Sigma\left(\Delta ; \theta_{0}\right)\right\|$ is finite, and $H(\Delta)$ is continuously-differentiable in $\Delta$ and the derivative is bounded. The functions $\nabla_{h} \zeta\left(\Delta ; \theta_{0}\right)$ exist and $\sup _{\Delta}\left\|\nabla_{h} \zeta\left(\Delta ; \theta_{0}\right)\right\|$ are finite for all integers $h \leq k$.

Note that

$$
\zeta\left(\Delta_{i} ; \theta_{0}\right)=\mathcal{E}^{*}\left[\eta_{i} \omega\left(x_{i 1}, x_{i 2}\right)\left\{\lambda_{i}\left(p_{i 1}+\iota_{i}\right)-\lambda_{i}\left(p_{i 2}+\iota_{i}\right)\right\} \mid \Delta_{i}\right] d\left(\Delta_{i}\right)
$$

and so, indeed, $\zeta\left(0 ; \theta_{0}\right)=0$.

The delta method then yields the asymptotic distribution of $\theta_{n}$. 
Theorem 3.1 (asymptotic Distribution). Let Assumptions 3.1-3.5 hold. Suppose that $\Sigma$ is positive definite, that $Q_{0}$ has full column rank, and that $V_{n} \stackrel{P}{\rightarrow} V_{0}$ for $V_{0}$ positive definite. Then $\sqrt{n \varsigma}\left\|\theta_{n}-\theta_{0}\right\|=O_{P}(1)$ and

$$
\sqrt{n \varsigma}\left(\theta_{n}-\theta_{0}\right) \stackrel{L}{\rightarrow} \mathcal{N}(0, \Upsilon), \quad \Upsilon \equiv\left(Q_{0}^{\prime} V_{0} Q_{0}\right)^{-1}\left(Q_{0}^{\prime} V_{0} \Sigma V_{0} Q_{0}\right)\left(Q_{0}^{\prime} V_{0} Q_{0}\right)^{-1}
$$

In particular, if $V_{n} \stackrel{P}{\rightarrow} \Sigma^{-1}$, then $\Upsilon=\left(Q_{0}^{\prime} \Sigma^{-1} Q_{0}\right)^{-1}$.

The asymptotic variance can be estimated using the plug-in estimates

$$
\begin{aligned}
Q_{n} & \equiv \frac{1}{n} \sum_{i=1}^{n} \frac{\omega\left(x_{i 1}, x_{i 2}\right)\left(\tau_{i 1}^{\prime}\left(\theta_{n}\right)-\tau_{i 2}^{\prime}\left(\theta_{n}\right)\right)^{\prime}}{\varsigma} \kappa\left(\frac{\widehat{\Delta}_{i}}{\varsigma}\right) s_{i 1} s_{i 2}, \\
\Sigma_{n} & \equiv \frac{1}{n} \sum_{i=1}^{n} \frac{\omega\left(x_{i 1}, x_{i 2}\right) \omega\left(x_{i 1}, x_{i 2}\right)^{\prime}\left(\tau_{i 1}\left(\theta_{n}\right)-\tau_{i 2}\left(\theta_{n}\right)\right)^{2}}{\varsigma} \kappa\left(\frac{\widehat{\Delta}_{i}}{\varsigma}\right)^{2} s_{i 1} s_{i 2},
\end{aligned}
$$

for $Q_{0}$ and $\Sigma$, respectively.

Theorem 3.2 (Inference). Let Assumptions 3.1-3.5 hold. Suppose that $\Sigma$ is positive definite and that $Q_{0}$ has full column rank. Then

$$
\Upsilon_{n} \stackrel{P}{\rightarrow} \Upsilon, \quad \Upsilon_{n} \equiv\left(Q_{n}^{\prime} V_{n} Q_{n}\right)^{-1}\left(Q_{n}^{\prime} V_{n} \Sigma_{n} V_{n} Q_{n}\right)\left(Q_{n}^{\prime} V_{n} Q_{n}\right)^{-1}
$$

if $V_{n} \stackrel{P}{\rightarrow} V_{0}$ for $V_{n}$ and $V_{0}$ positive definite.

Theorem 3.2 allows to conduct hypothesis tests on $\theta_{0}$ or transformations thereof, and also again implies validity of overidentification tests based on the optimally-weighted GMM criterion.

\subsection{Simulations}

As a numerical illustration we apply the fixed-effect estimator to a panel-data version of the Poisson model we used in the cross-sectional simulation exercise. To maximize the comparison between both sampling situations the data was generated in exactly the same way as before. Thus, For each of $n$ groups, two observations were generated from a Poisson distribution with conditional mean

$$
\mathcal{E}\left[y_{i j} \mid x_{i}, u_{i}\right]=\exp \left(c+x_{i j} \beta_{0}\right) u_{i}
$$

and all conditioning variables were drawn as before. The sample-selection process, too, was designed in the same way. The only design change, relative to the cross-sectional model, we consider is the sample size. Indeed, because, now, $\operatorname{Pr}\left[s_{i 1} s_{i 2}=1\right]=\operatorname{Pr}\left[s_{i 1}=1\right] \operatorname{Pr}\left[s_{i 2}=\right.$ $1]=\frac{1}{4}$, we double $n$ as to keep the effective sample size as before, on average. Smoothed maximum score was used as first-stage estimator. We again compare our estimator to the naive unweighted estimator based on the same set of moment conditions. Note that this estimator is $\sqrt{n}$-consistent when sample selection is exogenous. 
Table 3. One-step estimators

\begin{tabular}{|c|c|c|c|c|c|c|c|c|c|c|c|c|c|}
\hline \multirow[t]{2}{*}{$\rho$} & \multicolumn{2}{|l|}{$\bar{n} n$} & \multicolumn{4}{|c|}{ bias } & \multirow[b]{2}{*}{$\widehat{h}_{n}$} & \multicolumn{6}{|c|}{ " standard deviation } \\
\hline & $h_{n}$ & $\sim$ & 1 & 1.5 & 2.5 & 3 & & $\sim$ & 1 & 1.5 & 2.5 & 3 & $\widehat{h}_{n}$ \\
\hline 0 & 500 & .008 & .071 & .035 & .020 & .010 & .051 & .176 & .744 & .473 & .336 & .210 & .383 \\
\hline 0 & 1000 & .006 & .038 & .012 & .004 & .001 & .030 & .122 & .474 & .315 & .241 & .156 & .253 \\
\hline-0.5 & 500 & -.184 & .075 & .046 & .001 & -.090 & .049 & .155 & .679 & .432 & .306 & .198 & .446 \\
\hline-0.5 & 1000 & -.198 & .027 & .008 & -.013 & -.093 & .025 & .112 & .450 & .301 & .250 & .154 & .326 \\
\hline 0.5 & 500 & .257 & .073 & .051 & .078 & .168 & .037 & .195 & .760 & .475 & .323 & .225 & .330 \\
\hline 0.5 & 1000 & .254 & .058 & .047 & .064 & .146 & .025 & .136 & .522 & .337 & .260 & .168 & .244 \\
\hline \multirow{2}{*}{\multicolumn{2}{|c|}{$\rho$}} & \multicolumn{6}{|c|}{ standard error to standard deviation } & \multicolumn{6}{|c|}{ rejection frequency } \\
\hline & & $\sim$ & 1 & 1.5 & 2.5 & 3 & $\widehat{h}_{n}$ & $\sim$ & 1 & 1.5 & 2.5 & 3 & $\widehat{h}_{n}$ \\
\hline 0 & 500 & .956 & 1.226 & .875 & .921 & .971 & 1.115 & .059 & .076 & .062 & .068 & .050 & .049 \\
\hline 0 & 1000 & .979 & .943 & .954 & .967 & .987 & 1.149 & .046 & .057 & .060 & .056 & .053 & .034 \\
\hline-0.5 & 500 & .971 & .934 & .934 & .952 & .956 & 1.103 & .263 & .064 & .061 & .061 & .102 & .059 \\
\hline-0.5 & 1000 & .944 & .937 & .941 & .883 & .933 & .929 & .488 & .070 & .064 & .059 & .130 & .042 \\
\hline 0.5 & 500 & .957 & 1.064 & .875 & .957 & .952 & 1.260 & .255 & .075 & .069 & .061 & .108 & .043 \\
\hline 0.5 & 1000 & .969 & 1.262 & .924 & .927 & .965 & 1.326 & .477 & .064 & .056 & .055 & .129 & .038 \\
\hline
\end{tabular}

Table 4. Two-step estimators

\begin{tabular}{|c|c|c|c|c|c|c|c|c|c|c|c|c|c|}
\hline \multirow[t]{2}{*}{$\rho$} & \multirow[t]{2}{*}{ 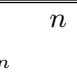 } & \multicolumn{5}{|c|}{ bias } & & \multicolumn{6}{|c|}{ standard deviation } \\
\hline & & $\sim$ & 1 & 1.5 & 2.5 & 3 & $\widehat{h}_{n}$ & $\sim$ & 1 & 1.5 & 2.5 & 3 & $\widehat{h}_{n}$ \\
\hline 0 & 500 & .013 & .162 & .064 & .029 & .012 & .123 & .171 & .769 & .464 & .317 & .206 & .638 \\
\hline 0 & 1000 & .006 & .089 & .036 & .017 & .005 & .061 & .117 & .481 & .311 & .223 & .150 & .462 \\
\hline-0.5 & 500 & -.148 & .145 & .059 & -.033 & -.115 & .101 & .151 & .698 & .435 & .285 & .192 & .636 \\
\hline-0.5 & 1000 & -.162 & .059 & .011 & -.050 & -.129 & .036 & .111 & .444 & .304 & .237 & .146 & .451 \\
\hline 0.5 & 500 & .235 & .173 & .122 & .155 & .208 & .123 & .188 & .733 & .478 & .327 & .225 & .585 \\
\hline 0.5 & 1000 & .230 & .118 & .099 & .137 & .199 & .069 & .132 & .510 & .334 & .253 & .168 & .431 \\
\hline$\rho$ & $n$ & \multicolumn{6}{|c|}{ standard error to standard deviation } & \multicolumn{6}{|c|}{ rejection frequency } \\
\hline \multicolumn{2}{|c|}{$h_{n}$} & $\sim$ & 1 & 1.5 & 2.5 & 3 & $\widehat{h}_{n}$ & $\sim$ & 1 & 1.5 & 2.5 & 3 & $\widehat{h}_{n}$ \\
\hline 0 & 500 & .940 & 1.100 & .837 & .891 & .949 & .781 & .070 & .086 & .071 & .069 & .046 & .073 \\
\hline 0 & 1000 & .982 & .877 & .927 & .959 & .981 & .748 & .044 & .066 & .063 & .056 & .049 & .066 \\
\hline-0.5 & 500 & .962 & .817 & .859 & .916 & .935 & .695 & .211 & .080 & .075 & .073 & .131 & .113 \\
\hline-0.5 & 1000 & .930 & .908 & .894 & .849 & .921 & .743 & .396 & .071 & .067 & .080 & .203 & .097 \\
\hline 0.5 & 500 & .938 & 1.051 & .843 & .898 & .936 & .857 & .254 & .082 & .082 & .080 & .143 & .097 \\
\hline 0.5 & 1000 & .956 & 1.733 & .905 & .902 & .943 & .810 & .436 & .067 & .065 & .080 & .232 & .088 \\
\hline
\end{tabular}

Tables 3 and 4 have the same layout as before. The performance of the estimator, too, is in line with the cross-sectional case and so we can be brief in our description of the tables. The naive estimator is again heavily biased when selection into the sample is endogenous. Our approach tends to deliver estimators with relatively small bias, except in some cases when the bandwidth is large. Eventhough the effective sample size is quite small, the ratio of the estimated standard error to the standard deviation is reasonably close to unity across the designs, so that inference based on the asymptotic distribution theory derived above may be reliable. A look at the rejection frequencies confirms this. 


\section{Acknowledgments}

A previous version of this paper circulated under the title 'Simple estimators for count data models with sample selection'. I am grateful to Han Hong, an associate editor, and two referees for very constructive comments.

\section{Appendix A. Proof of Theorems 2.1 and 2.2}

Notation. The following notation will be used. Let $\xi_{i j}(\theta) \equiv \omega\left(x_{i}, x_{j}\right)\left(\tau_{i}(\theta)-\tau_{j}(\theta)\right)$. Then

$$
q_{n}(\theta) \equiv\left(\begin{array}{l}
n \\
2
\end{array}\right)^{-1} \sum_{i=1}^{n} \sum_{i<j} \frac{\xi_{i j}(\theta)}{\varsigma} \kappa\left(\frac{p_{i}-p_{j}}{\varsigma}\right) s_{i} s_{j}
$$

Also, $q_{0}(\theta) \equiv \mathcal{E}\left[\zeta_{i}\left(p_{i} ; \theta\right)\right]$. Note that $q_{n}(\theta)$ is an infeasible version of $\widehat{q}_{n}(\theta)$ where the kernel weight depends on the true $p_{i}$ and that, under our assumptions, $q_{0}(\theta)$ is the limit of this empirical moment condition, i.e, $q_{0}(\theta)=\lim _{n \uparrow+\infty} \mathscr{E}\left[q_{n}(\theta)\right]$, as will be verified below. We let $\xi_{i j}^{\prime}(\theta) \equiv \omega\left(x_{i}, x_{j}\right)\left(\tau_{i}^{\prime}(\theta)-\tau_{j}^{\prime}(\theta)\right)^{\prime}$ and write $\widehat{Q}_{n}(\theta), Q_{n}(\theta)$, and $Q_{0}(\theta)$ for the Jacobian matrices associated with $\widehat{q}_{n}(\theta), q_{n}(\theta)$, and $q_{0}(\theta)$, respectively.

Consistency. Given the regularity conditions in Assumption 2.1 and the fact that $V_{n} \stackrel{P}{\rightarrow} V_{0}$ by construction, it suffices to show that

$$
\sup _{\theta \in \Theta}\left\|\widehat{q}_{n}(\theta)-q_{0}(\theta)\right\|=o_{P}(1)
$$

Consistency will then follow from Theorem 2.1 of Newey and McFadden (1994). Note that the moment conditions in Assumption 2.4 imply that there exist $a>0$ and $C_{n}=O_{P}(1)$ such that, for all pairs $\theta_{1}, \theta_{2} \in \Theta, s_{i} s_{j}\left\|\xi_{i j}\left(\theta_{1}\right)-\xi_{i j}\left(\theta_{2}\right)\right\| \leq C_{n}\left\|\theta_{1}-\theta_{2}\right\|^{a}$. Together with the boundedness of the kernel function, this implies that $\left\|\widehat{q}_{n}\left(\theta_{1}\right)-\widehat{q}_{n}\left(\theta_{2}\right)\right\| \leq O_{P}(1)\left\|\theta_{1}-\theta_{2}\right\|^{a}$. Therefore, the uniform convergence result will follow if we can show that $\widehat{q}_{n}(\theta) \stackrel{P}{\rightarrow} q_{0}(\theta)$ for all $\theta \in \Theta$; see Lemma 2.9 in Newey and McFadden (1994). To do so, we use the triangle inequality to get the bound

$$
\left\|\widehat{q}_{n}(\theta)-q_{0}(\theta)\right\| \leq\left\|\widehat{q}_{n}(\theta)-q_{n}(\theta)\right\|+\left\|q_{n}(\theta)-q_{0}(\theta)\right\|
$$

for each $\theta \in \Theta$, and establish the pointwise convergence in probability of each of the terms on the right-hand side.

To tackle the estimation noise in the $\widehat{p}_{i}$, use the continuity of the kernel function and boundedness of its derivative stated in Assumption 2.3 to validate a mean-value expansion around $\gamma_{0}$. This shows that $\left\|\widehat{q}_{n}(\theta)-q_{n}(\theta)\right\|$ is bounded by

$$
\frac{\sup _{\varepsilon}\left|\kappa^{\prime}(\varepsilon)\right|\left(\begin{array}{c}
n \\
2
\end{array}\right)^{-1} \sum_{i=1}^{n} \sum_{j \neq i} s_{i} s_{j}\left\|\xi_{i j}(\theta)\right\|\left\|z_{i}\right\|}{\varsigma^{2}}\left\|\gamma_{n}-\gamma_{0}\right\|=O_{P}\left(\frac{1}{\varsigma^{2} \sqrt{n}}\right) .
$$


The first transition follows from the symmetry of the functions $\xi_{i j}(\theta)$ and $\kappa$, and the second transition follows from the finite-moment requirements in Assumption 2.4 and by the $\sqrt{n}$ consistency of $\gamma_{n}$ stated in Assumption 2.2. Assumption 2.3 then ensures the bandwidth sequence to be so that this term is $o_{P}(1)$.

To see that $\left\|q_{n}(\theta)-q_{0}(\theta)\right\|=o_{P}(1)$, first note that $\left\|q_{n}-\mathscr{E}\left[q_{n}(\theta)\right]\right\|=o_{P}(1)$. Indeed, by the Cauchy-Schwarz inequality, $\mathscr{E}^{*}\left[\left\|\xi_{i j}(\theta)\right\|^{2}\right] \leq \sqrt{2 \mathscr{E}^{*}\left[\left\|\omega\left(x_{i}, x_{j}\right)\right\|^{4}\right] \mathcal{E}^{*}\left[\left|\tau_{i}(\theta)\right|^{4}\right]}$, which is $O_{P}(1)$ by Assumption 2.3. Furthermore, by the boundedness of the kernel function stated in Assumption 2.4, $\mathscr{E}\left[q_{n}(\theta)\right]$ exists and

$$
\mathscr{E}^{*}\left[\left\|\frac{\xi_{i j}(\theta)}{\varsigma} \kappa\left(\frac{p_{i}-p_{j}}{\varsigma}\right)\right\|^{2}\right] \leq \frac{\mathscr{E}^{*}\left[\left\|\xi_{i j}(\theta)\right\|^{2}\right] \sup _{\varepsilon}|\kappa(\varepsilon)|}{\varsigma^{2}}=O_{P}\left(\frac{1}{\varsigma^{2}}\right)=o(n),
$$

with the last transition following from the rate conditions on the bandwidth sequence. Lemma 3.1 of Powell, Stock, and Stoker (1989) then provides the appropriate law of large numbers. Next,

$$
\mathscr{E}\left[\frac{\xi_{i j}(\theta)}{\varsigma} \kappa\left(\frac{p_{i}-p_{j}}{\varsigma}\right) s_{i} s_{j}\right]=\mathcal{E}\left[\int_{-\infty}^{+\infty} \frac{\zeta_{i}(p ; \theta)}{\varsigma} \kappa\left(\frac{p_{i}-p}{\varsigma}\right) \mathrm{d} p\right] \rightarrow \mathcal{E}\left[\zeta_{i}\left(p_{i} ; \theta\right)\right],
$$

by a dominated-convergence argument validated through the dominance condition on $\zeta_{i}(p ; \theta)$ in Assumption 2.4, and so we have that $\left.\left\|\mathscr{E}\left[q_{n}(\theta)\right]-q_{0}(\theta)\right\|=o_{(} 1\right)$. By the triangle inequality,

$$
\left\|q_{n}(\theta)-q_{0}(\theta)\right\| \leq\left\|q_{n}-\mathscr{E}\left[q_{n}(\theta)\right]\right\|+\left\|\mathscr{E}\left[q_{n}(\theta)\right]-q_{0}(\theta)\right\|
$$

and the result follows.

Combining (A.1) through (A.3) then implies that $\left\|\widehat{q}_{n}(\theta)-q_{0}(\theta)\right\|=o_{P}(1)$ for each $\theta \in \Theta$, which is what we wanted to show.

Projection of the empirical moment. The main step in deriving the limit distribution of $\theta_{n}$ is showing that

$$
\widehat{q}_{n}\left(\theta_{0}\right)=\frac{2}{n} \sum_{i=1}^{n} \sigma_{i}+o_{P}(1 / \sqrt{n}) .
$$

To do so, note that a second-order expansion of $\widehat{q}_{n}(\theta)$ around the first-stage estimator gives

$$
\widehat{q}_{n}(\theta)-q_{n}(\theta)=\left(\begin{array}{l}
n \\
2
\end{array}\right)^{-1} \sum_{i=1}^{n} \sum_{j \neq i} \frac{\xi_{i j}(\theta)}{\varsigma} \kappa^{\prime}\left(\frac{\left(z_{i}-z_{j}\right)^{\prime} \gamma_{0}}{\varsigma}\right) \frac{z_{i}^{\prime}\left(\gamma_{n}-\gamma_{0}\right)}{\varsigma} s_{i} s_{j}+R_{n}
$$

for a remainder term $R_{n}$ which will be shown to be asymptotically negligible. To show (A.4) we will proceed in two steps. The first is to work out the expression for $\widehat{q}_{n}\left(\theta_{0}\right)-q_{n}\left(\theta_{0}\right)$ above up to $o_{P}(1 / \sqrt{n})$, the second is to approximate the $U$-statistic $q_{n}\left(\theta_{0}\right)$ by its projection and to show that the approximation error is $o_{P}(1 / \sqrt{n})$.

To quantify the impact of first-stage estimation error, first note that $R_{n}$ is bounded by

$$
\frac{\frac{1}{2}\left(\begin{array}{c}
n \\
2
\end{array}\right)^{-1} \sum_{i=1}^{n} \sum_{j \neq i} s_{i} s_{j}\left\|\xi_{i j}(\theta)\right\|\left\|z_{i}\right\|^{2} \sup _{\varepsilon}\left|\kappa^{\prime \prime}(\varepsilon)\right|}{\varsigma^{3}}\left\|\gamma_{n}-\gamma_{0}\right\|^{2}=O_{P}\left(\frac{1}{\varsigma^{3} n}\right)
$$


by the conditions on the kernel in Assumption 2.3, the moment conditions in Assumption 2.4, and the $\sqrt{n}$-convergence rate of $\left\|\gamma_{n}-\gamma_{0}\right\|$ in Assumption 2.2. Furthermore, because $\varsigma \propto n^{-r}$ for some $r<\frac{1}{6}$, this term is $o_{P}(1 / \sqrt{n})$ and, thus, is asymptotically negligible. Replacing $\gamma_{n}-\gamma_{0}$ by its influence-function expression in (B.2) gives

$$
\widehat{q}_{n}(\theta)-q_{n}(\theta)=\frac{1}{3}\left(\begin{array}{l}
n \\
3
\end{array}\right)^{-1} \sum_{i=1}^{n} \sum_{j \neq i} \sum_{k \neq i, j} \frac{\xi_{i j}(\theta) s_{i} s_{j}}{\varsigma} \kappa^{\prime}\left(\frac{\left(z_{i}-z_{j}\right)^{\prime} \gamma_{0}}{\varsigma}\right) \frac{z_{i}^{\prime} \psi_{k}}{\varsigma}+o_{P}\left(\frac{1}{\sqrt{n}}\right),
$$

where we ignore terms for which either $k=i$ or $k=j$, as they are asymptotically negligible, and rescale appropriately; the effect of this rescaling is asymptotically negligible. Indeed, the contribution of terms with $k=i$, for example, is bounded by

$$
\left(\begin{array}{l}
n \\
2
\end{array}\right)^{-1} \sum_{i=1}^{n} \sum_{j \neq i} \frac{1}{n}\left\|\frac{\xi_{i j}(\theta) s_{i} s_{j}}{\varsigma} \kappa^{\prime}\left(\frac{\left(z_{i}-z_{j}\right)^{\prime} \gamma_{0}}{\varsigma}\right) \frac{z_{i}^{\prime} \psi_{i}}{\varsigma}\right\|=O_{P}\left(\frac{1}{\varsigma^{2} n}\right)=o_{P}\left(\frac{1}{\sqrt{n}}\right),
$$

which follows from Assumptions 2.2, 2.3, and 2.4. A symmetrization argument then allows writing (B.2) as a third-order $U$-statistic whose second moment is $o(n)$; this can be verified using the same arguments as were used to show consistency. Therefore, by Lemma 3.1 of Powell, Stock, and Stoker (1989), $\widehat{q}_{n}(\theta)-q_{n}(\theta)$ differs from its projection by a term that is $o_{P}(1 / \sqrt{n})$. The projection itself equals

$$
\frac{2}{3} \mathcal{E}\left[h_{i}(\theta)\right]-\frac{2}{n} \sum_{i=1}^{n}\left\{h_{i}(\theta)-\mathcal{E}\left[h_{i}(\theta)\right]\right\}, \quad h_{i}(\theta) \equiv \mathscr{E}\left[\frac{\xi_{j k}(\theta) z_{j}^{\prime}}{\varsigma^{2}} \kappa^{\prime}\left(\frac{p_{j}-p_{k}}{\varsigma}\right) s_{j} s_{k}\right] \psi_{i} .
$$

Now, by a change-of-variable in the first step and integration by parts in the second step,

$$
\begin{aligned}
h_{i}(\theta) & =\mathcal{E}\left[\frac{\int_{-\infty}^{+\infty} \zeta_{j}\left(p_{j}-\varsigma \eta ; \theta\right) z_{j}^{\prime} \kappa^{\prime}(\eta) \mathrm{d} \eta}{\varsigma}\right] \psi_{i} \\
& =\mathcal{E}\left[\frac{\left.\zeta_{j}\left(p_{j}-\varsigma \eta ; \theta\right) z_{j}^{\prime} \kappa(\eta)\right|_{-\infty} ^{+\infty}}{\varsigma}\right] \psi_{i}-\mathcal{E}\left[\int_{-\infty}^{+\infty} \nabla_{1} \zeta_{j}\left(p_{j}-\varsigma \eta ; \theta\right) \kappa(\eta) \mathrm{d} \eta z_{j}^{\prime}\right] \psi_{i} .
\end{aligned}
$$

On evaluating in $\theta_{0}$ the first right-hand side term is zero, being bounded in magnitude by

$$
\mathcal{E}\left[\left\|\frac{\left.\zeta_{j}\left(p_{j}-\varsigma \eta ; \theta_{0}\right) z_{j}^{\prime} \kappa(\eta)\right|_{-\infty} ^{+\infty}}{\varsigma}\right\|\right] \psi_{i} \leq \frac{\left.\sqrt{\mathcal{E}\left[\left\|z_{j}\right\|^{2}\right] \mathcal{E}\left[\sup _{p}\left\|\zeta_{j}\left(p ; \theta_{0}\right)\right\|^{2}\right]} \kappa(\eta)\right|_{-\infty} ^{+\infty}}{\varsigma} \psi_{i}=0
$$

because the relevant moments exist and $\kappa(\varepsilon)-\kappa(-\varepsilon)=0$ for any $\varepsilon>0$. Further, because $\kappa$ is a $k$ th-order kernel, a $k$ th-order Taylor expansion of $\nabla_{1} \zeta_{j}\left(p_{j}-\varsigma \eta ; \theta_{0}\right)$ around $\eta=0$ yields

$\mathcal{E}\left[\int_{-\infty}^{+\infty} \nabla_{1} \zeta_{j}\left(p_{j}-\varsigma \eta ; \theta\right) \kappa(\eta) \mathrm{d} \eta z_{j}^{\prime}\right]=\mathcal{E}\left[\nabla_{1} \zeta_{j}\left(p_{j} ; \theta_{0}\right) z_{j}^{\prime}+\frac{\int_{-\infty}^{+\infty} \nabla_{k+1} \zeta_{j}\left(* ; \theta_{0}\right) \eta^{k} \kappa(\eta) \mathrm{d} \eta z_{j}^{\prime}}{\varsigma^{-k} k !}\right]$,

where $*$ lies between $p_{j}-\varsigma \eta$ and $p_{j}$. Invoking Assumptions 2.3 and 2.5 and applying a dominated-convergence argument to the remainder term gives

$$
\left\|\mathcal{E}\left[\frac{\int_{-\infty}^{+\infty} \nabla_{k+1} \zeta_{j}\left(* ; \theta_{0}\right) \eta^{k} \kappa(\eta) \mathrm{d} \eta z_{j}^{\prime}}{k !}\right]\right\| \leq C \frac{\sqrt{\mathcal{E}\left[\left\|z_{j}\right\|^{2}\right] \mathcal{E}\left[\sup _{p}\left\|\nabla_{k+1} \zeta_{j}\left(p ; \theta_{0}\right)\right\|^{2}\right]}}{k !}=O_{P}(1)
$$


for $C \equiv \int_{-\infty}^{+\infty}\left|\varepsilon^{k}\right||\kappa(\varepsilon)| \mathrm{d} \varepsilon$. Because Assumption 2.3 implies that $\varsigma^{k}=o_{P}(1 / \sqrt{n})$, we obtain

$$
h_{i}\left(\theta_{0}\right)=-\mathcal{E}\left[\nabla_{1} \zeta_{j}\left(p_{j} ; \theta_{0}\right) z_{j}^{\prime}\right] \psi_{i}+o_{P}(1 / \sqrt{n}) .
$$

Moreover,

$$
\widehat{q}_{n}\left(\theta_{0}\right)-q_{n}\left(\theta_{0}\right)=-\frac{2}{n} \sum_{i=1}^{n} \mathcal{E}\left[\nabla_{1} \zeta_{j}\left(p_{j} ; \theta_{0}\right) z_{j}^{\prime}\right] \psi_{i}+o_{P}\left(\frac{1}{\sqrt{n}}\right),
$$

because $\mathcal{E}\left[\psi_{i}\right]=0$.

Now turn to the projection of $q_{n}\left(\theta_{0}\right)$. By the arguments in the proof of consistency, we may again invoke Lemma 3.1 of Powell, Stock, and Stoker (1989) to justify that $q_{n}\left(\theta_{0}\right)$ equals its projection

$$
\mathcal{E}\left[g_{i}\left(\theta_{0}\right)\right]+\frac{2}{n} \sum_{i=1}^{n}\left\{g_{i}\left(\theta_{0}\right)-\mathcal{E}\left[g_{i}\left(\theta_{0}\right)\right]\right\}, \quad g_{i}(\theta) \equiv \mathcal{E}\left[\frac{\xi_{i j}(\theta)}{\varsigma} \kappa\left(\frac{p_{i}-p_{j}}{\varsigma}\right) s_{i} s_{j}\right],
$$

up to a term that is $o_{P}(1 / \sqrt{n})$. By using a $k$ th-order Taylor expansion and proceeding as before, Assumption 2.5 yields $g_{i}\left(\theta_{0}\right)=\zeta_{i}\left(p_{i} ; \theta_{0}\right)+o_{P}(1 / \sqrt{n})$, and so

$$
q_{n}\left(\theta_{0}\right)=\frac{2}{n} \sum_{i=1}^{n} \zeta_{i}\left(p_{i} ; \theta_{0}\right)+o_{P}\left(\frac{1}{\sqrt{n}}\right)
$$

because $\mathcal{E}\left[g_{i}\left(\theta_{0}\right)\right]=o_{P}(1 / \sqrt{n})$.

Combine (A.6) and (A.7) to see that they imply (A.4).

Convergence of the Jacobian matrix. The same steps as those used to prove consistency will yield pointwise convergence of the Jacobian matrix, i.e.,

$$
\left\|\widehat{Q}_{n}(\theta)-Q_{0}(\theta)\right\| \leq\left\|\widehat{Q}_{n}(\theta)-Q_{n}(\theta)\right\|+\left\|Q_{n}(\theta)-Q_{0}(\theta)\right\|=o_{P}(1)
$$

for al $\theta \in \Theta$. Also, $\widehat{Q}_{n}(\theta)$ is continuous in $\theta$ and $s_{i} s_{j}\left\|\widehat{Q}_{n}\left(\theta_{1}\right)-\widehat{Q}_{n}\left(\theta_{2}\right)\right\| \leq O_{P}(1)\left\|\theta_{1}-\theta_{2}\right\|^{a}$ for some $a>0$, and so Lemma 2.9 in Newey and McFadden (1994) will again imply the uniform-convergence result sought for. For the first right-hand side term in (A.8), observe that

$$
\left\|\widehat{Q}_{n}(\theta)-Q_{n}(\theta)\right\| \leq \frac{1}{\varsigma^{2}}\left(\begin{array}{l}
n \\
2
\end{array}\right)^{-1} \sum_{i=1}^{n} \sum_{i<j} s_{i} s_{j}\left\|\xi_{i j}^{\prime}(\theta)\right\|\left\|z_{i}\right\|\left\|\gamma_{n}-\gamma_{0}\right\|=O_{P}\left(\frac{1}{\varsigma^{2} \sqrt{n}}\right)=o_{P}(1) .
$$

For the second right-hand side term, use the triangle inequality to get

$$
\left\|Q_{n}(\theta)-Q_{0}(\theta)\right\| \leq\left\|Q_{n}(\theta)-\mathscr{E}\left[Q_{n}(\theta)\right]\right\|+\left\|\mathscr{E}\left[Q_{n}(\theta)\right]-Q_{0}(\theta)\right\|=o_{P}(1) .
$$

Because $\mathscr{E}\left[Q_{n}(\theta)\right]$ exists and

$$
\mathscr{E}^{*}\left[\left\|\frac{\xi^{\prime}(\theta)}{\varsigma} \kappa\left(\frac{p_{i}-p_{j}}{\varsigma}\right)\right\|^{2}\right]=o(n)
$$


Lemma 3.1 of Powell, Stock, and Stoker (1989) establishes that $\left\|Q_{n}(\theta)-\mathscr{E}\left[Q_{n}(\theta)\right]\right\|=o_{P}(1)$. Also

$$
\mathscr{E}\left[\frac{\xi^{\prime}(\theta)}{\varsigma} \kappa\left(\frac{p_{i}-p_{j}}{\varsigma}\right) s_{i} s_{j}\right]=\mathcal{E}\left[\int_{-\infty}^{+\infty} \frac{\zeta_{i}^{\prime}(p ; \theta)}{\varsigma} \kappa\left(\frac{p_{i}-p}{\varsigma}\right) \mathrm{d} p\right] \rightarrow \mathcal{E}\left[\zeta_{i}^{\prime}\left(p_{i} ; \theta\right)\right],
$$

and so $\left\|\mathscr{E}\left[Q_{n}(\theta)\right]-Q_{0}(\theta)\right\|=o_{P}(1)$. Put together this verifies (A.8) and yields uniform convergence.

Asymptotic distribution. By continuity of $\widehat{q}_{n}(\theta)$ in $\theta$, in tandem with (A.4) and the uniform convergence of $\widehat{Q}_{n}(\theta)$ to $Q_{0}(\theta)$ on $\Theta$, an expansion of the first-order conditions of the GMM minimization problem yields

$$
\sqrt{n}\left(\theta_{n}-\theta_{0}\right)=-\left(Q_{0}^{\prime} V_{0} Q_{0}\right)^{-1} Q_{0}^{\prime} V_{0} \frac{2}{\sqrt{n}} \sum_{i=1}^{n} \sigma_{i}+o_{P}(1)
$$

where, recall $V_{n} \stackrel{P}{\rightarrow} V_{0}$. Now, Assumptions 2.2 and 2.5 imply that $\operatorname{var}\left[\sigma_{i}\right]=\Sigma<+\infty$ while, clearly, $\mathcal{E}\left[\sigma_{i}\right]=0$. Hence, $\theta_{n}$ is asymptotically linear, and $\sqrt{n}\left(\theta_{n}-\theta_{0}\right) \stackrel{L}{\rightarrow} \mathcal{N}(0, \Upsilon)$. When $V_{n}$ is so that $V_{0}=\Sigma^{-1}$, a calculation verifies that $\Upsilon=4\left(Q_{0}^{\prime} \Sigma^{-1} Q_{0}\right)^{-1}$.

Inference. Because $V_{n} \stackrel{P}{\rightarrow} V_{0}$ by assumption, it suffices to show that (i) $\left\|Q_{n}-Q_{0}\right\|=o_{P}(1)$ and that (ii) $\left\|\Sigma_{n}-\Sigma\right\|=o_{P}(1)$.

To see that (i) holds, note that we have $\left\|\widehat{Q}_{n}\left(\theta_{n}\right)-\widehat{Q}_{n}\left(\theta_{0}\right)\right\|=O_{P}(1)\left\|\theta_{n}-\theta_{0}\right\|^{a}$ for some $a>0$ from the argument above, and observe that $Q_{n}=\widehat{Q}_{n}\left(\theta_{n}\right)$. Further, because we have shown that $\left\|\theta_{n}-\theta_{0}\right\|=O_{P}(1 / \sqrt{n}), Q_{n}=\widehat{Q}_{n}\left(\theta_{0}\right)+o_{P}(1)$. The results then follows from the pointwise convergence result in (A.8).

To see that (ii) holds, first note that it is implied by $n^{-1} \sum_{i=1}^{n}\left\|\widehat{\sigma}_{i}-\sigma_{i}\right\|^{2}=o_{P}(1)$. Now,

$$
\frac{1}{n} \sum_{i=1}^{n}\left\|\widehat{\sigma}_{i}-\sigma_{i}\right\|^{2} \leq \frac{1}{n} \sum_{i=1}^{n}\left\{\left\|\widehat{\zeta}_{i}-\zeta_{i}\right\|^{2}+\left\|H_{0}\right\|^{2}\left\|\widehat{\psi}_{i}-\psi_{i}\right\|^{2}+\left\|\psi_{i}\right\|^{2}\left\|H_{n}-H_{0}\right\|^{2}\right\}+R_{n}
$$

where $R_{n}$ captures lower-order terms and $\zeta_{i} \equiv \zeta_{i}\left(p_{i} ; \theta_{0}\right)$. All the dominant right-hand side contributions will be $o_{P}(1)$ provided we can show that $\left\|H_{n}-H_{0}\right\|=o_{P}(1)$ and $n^{-1} \sum_{i=1}^{n}\left\|\widehat{\zeta}_{i}-\zeta_{i}\right\|^{2}=o_{P}(1)$, as we assume that $\widehat{\psi}_{i}$ is so that $n^{-1} \sum_{i=1}^{n}\left\|\widehat{\psi}_{i}-\psi_{i}\right\|^{2}=o_{P}(1)$. Start with the convergence of $H_{n}$. By an analogous reasoning as for $Q_{n}$, and a further expansion around $\gamma_{0}$,

$$
H_{n}=\left(\begin{array}{l}
n \\
2
\end{array}\right)^{-1} \sum_{i=1}^{n} \sum_{j \neq i} \frac{\xi_{i j}\left(\theta_{0}\right) s_{i} s_{j}}{\varsigma} \kappa^{\prime}\left(\frac{p_{i}-p_{j}}{\varsigma}\right) \frac{z_{i}^{\prime}}{\varsigma}+o_{P}(1)
$$

The summand on the right-hand side is already known to satisfy the conditions for the law of large numbers to apply. Further,

$$
\mathscr{E}\left[\frac{\xi_{i j}\left(\theta_{0}\right) s_{i} s_{j}}{\varsigma} \kappa^{\prime}\left(\frac{p_{i}-p_{j}}{\varsigma}\right) \frac{z_{i}^{\prime}}{\varsigma}\right] \rightarrow H_{0},
$$


as was shown in the derivation of (A.6), and so $\left\|H_{n}-H_{0}\right\|=o_{P}(1)$. Also, again using similar arguments,

$$
\frac{1}{n} \sum_{i=1}^{n}\left\|\widehat{\zeta}_{i}-\widetilde{\zeta}_{i}\right\|^{2}=o_{P}(1), \quad \widetilde{\zeta}_{i} \equiv \frac{1}{n-1} \sum_{j \neq i} \frac{\omega\left(x_{i}, x_{j}\right)\left(\tau_{i}\left(\theta_{0}\right)-\tau_{j}\left(\theta_{0}\right)\right)}{\varsigma} \kappa\left(\frac{p_{i}-p_{j}}{\varsigma}\right) s_{i} s_{j},
$$

is established. Finally, from the proof of Theorem 3.4 in Powell, Stock, and Stoker (1989) we immediately have

$$
\mathcal{E}\left[\left\|\widetilde{\zeta}_{i}-\bar{\zeta}_{i}\right\|^{2}\right]=O\left(\frac{1}{\varsigma^{3} n}\right)=o(1), \quad \bar{\zeta}_{i} \equiv \int_{-\infty}^{+\infty} \frac{\zeta_{i}\left(p ; \theta_{0}\right)}{\varsigma} \kappa\left(\frac{p_{i}-p}{\varsigma}\right) \mathrm{d} p,
$$

while the smoothness and dominance conditions in Assumption 2.5 imply that $\mathcal{E}\left[\left\|\bar{\zeta}_{i}-\zeta_{i}\right\|^{2}\right]$ equals

$$
\mathcal{E}\left[\left(\int_{-\infty}^{+\infty}\left\|\zeta_{i}\left(p_{i}-\varsigma \eta ; \theta_{0}\right)-\zeta_{i}\left(p_{i} ; \theta_{0}\right)\right\| \kappa(\eta) \mathrm{d} \eta\right)^{2}\right] \leq \varsigma \mathcal{E}\left[\underset{p}{\sup }\left\|\nabla_{1} \zeta_{i}\left(p ; \theta_{0}\right)\right\|^{2}\right] C^{2}=O(\varsigma),
$$

for $C \equiv \int_{-\infty}^{+\infty}|\varepsilon||\kappa(\varepsilon)| \mathrm{d} \varepsilon$, which is $o(1)$. Therefore, $n^{-1} \sum_{i=1}^{n}\left\|\widetilde{\zeta}_{i}-\overline{\zeta_{i}}\right\|^{2}=o_{P}(1)$ and $n^{-1} \sum_{i=1}^{n}\left\|\bar{\zeta}_{i}-\zeta_{i}\right\|^{2}=o_{P}(1)$ by the law of large numbers, and $n^{-1} \sum_{i=1}^{n}\left\|\widetilde{\zeta}_{i}-\zeta_{i}\right\|^{2}=o_{P}(1)$ follows. Therefore, (ii) has been shown and the proof is complete.

\section{Appendix B. Proof of Theorems 3.1 and 3.2}

Notation. The following notation will be used. Let $\xi_{i}(\theta) \equiv \omega\left(x_{i 1}, x_{i 2}\right)\left(\tau_{i 1}(\theta)-\tau_{i 2}(\theta)\right)$ and $s_{i} \equiv s_{i 1} s_{i 2}$. Then

$$
q_{n}(\theta) \equiv \frac{1}{n} \sum_{i=1}^{n} \frac{\xi_{i}(\theta)}{\varsigma} \kappa\left(\frac{\Delta_{i}}{\varsigma}\right) s_{i}
$$

Also, $q_{0}(\theta) \equiv \zeta(0 ; \theta)$. Note that $q_{n}(\theta)$ is the empirical moment condition that takes $\gamma_{0}$ as known, and that $q_{0}(\theta)$ is the large- $n$ limit of this function. Similarly, we again let $\xi_{i}^{\prime}(\theta) \equiv \omega\left(x_{i 1}, x_{i 2}\right)\left(\tau_{i 1}^{\prime}(\theta)-\tau_{i 2}^{\prime}(\theta)\right)^{\prime}$ and write $\widehat{Q}_{n}(\theta), Q_{n}(\theta)$, and $Q_{0}(\theta)$ for the Jacobian matrices associated with $\widehat{q}_{n}(\theta), q_{n}(\theta)$, and $q_{0}(\theta)$, respectively.

Consistency. We follow the same steps as in Appendix A to establish consistency of $\theta_{n}$ in the panel-data case. For any fixed $\theta \in \Theta$, by a mean-value expansion around $\gamma_{0}$, we have that

$$
\left\|\widehat{q}_{n}(\theta)-q_{n}(\theta)\right\| \leq \frac{\sup _{\varepsilon}\left|\kappa^{\prime}(\varepsilon)\right| \frac{1}{n} \sum_{i=1}^{n} s_{i}\left\|\xi_{i}(\theta)\right\|\left\|z_{i 1}-z_{i 2}\right\|}{\varsigma^{2}}\left\|\gamma_{n}-\gamma_{0}\right\|=O_{P}\left(\frac{1}{n^{s-2 r}}\right),
$$

by the the moment conditions in Assumption 3.4 and the convergence rate of the first-stage estimator. Because we set $r<s / 2$, this term converges to zero in probability. Also, by a standard law of large numbers, $\left\|q_{n}(\theta)-\mathcal{E}\left[q_{n}(\theta)\right]\right\|$ is

$$
\left\|\frac{1}{n} \sum_{i=1}^{n} \frac{\xi_{i}(\theta)}{\varsigma} \kappa\left(\frac{p_{i 1}-p_{i 2}}{\varsigma}\right) s_{i}-\mathcal{E}\left[\frac{\xi_{i}(\theta)}{\varsigma} \kappa\left(\frac{p_{i 1}-p_{i 2}}{\varsigma}\right) s_{i}\right]\right\|=O_{P}\left(\frac{1}{\sqrt{n}}\right) .
$$


Further, $\left\|\mathcal{E}\left[q_{n}(\theta)-q_{0}(\theta)\right]\right\|=o(1)$ because

$$
\mathcal{E}\left[q_{n}(\theta)\right]=\mathcal{E}\left[\frac{\xi_{i}(\theta)}{\varsigma} \kappa\left(\frac{p_{i 1}-p_{i 2}}{\varsigma}\right) s_{i}\right]=\int_{-\infty}^{+\infty} \frac{\zeta(\Delta ; \theta)}{\varsigma} \kappa\left(\frac{\Delta}{\varsigma}\right) \mathrm{d} \Delta \rightarrow q_{0}(\theta)
$$

by a standard bounded-convergence argument, validated by the conditions in Assumption 3.4. Therefore, $\left\|\widehat{q}_{n}(\theta)-q_{0}(\theta)\right\|=o_{p}(1)$. The regularity conditions in Assumption 3.1 then ensure the remaining conditions for Lemma 2.9 in Newey and McFadden (1994) to apply, and consistency follows.

Expansion of the empirical moment. The limit distribution of $\theta_{n}$ is the same as the limit distribution of the infeasible estimator $\arg \max _{\theta \in \Theta} q_{n}(\theta)^{\prime} V_{n} q_{n}(\theta)$. To show this it suffices to show that

$$
\sqrt{n \varsigma}\left\{\widehat{q}_{n}\left(\theta_{0}\right)-q_{n}\left(\theta_{0}\right)\right\}=o_{P}(1),
$$

that is, that the contribution of the estimation noise introduced by the first-stage estimator of $\gamma_{0}$ is asymptotically negligible. To do so, consider a second-order expansion around $\gamma_{0}$ to get

$$
\begin{aligned}
\widehat{q}_{n}\left(\theta_{0}\right)-q_{n}\left(\theta_{0}\right) & =\frac{1}{n} \sum_{i=1}^{n} s_{i} \frac{\xi_{i}\left(\theta_{0}\right)}{\varsigma} \kappa^{\prime}\left(\frac{\Delta_{i}}{\varsigma}\right) \frac{\left(z_{i 1}-z_{i 2}\right)^{\prime}}{\varsigma}\left(\gamma_{n}-\gamma_{0}\right) \\
& +\frac{1}{n} \sum_{i=1}^{n} s_{i} \frac{\xi_{i}\left(\theta_{0}\right)}{\varsigma} \kappa^{\prime \prime}(*) \frac{\left(z_{i 1}-z_{i 2}\right)^{\prime}}{\varsigma}\left(\gamma_{n}-\gamma_{0}\right)\left(\gamma_{n}-\gamma_{0}\right)^{\prime} \frac{\left(z_{i 1}-z_{i 2}\right)}{\varsigma},
\end{aligned}
$$

where $*$ is as before. As shown in the proof to consistency above, the expectation of the first term on the right-hand side in (B.2) exists. With $H\left(\Delta_{i}\right)=\mathcal{E}^{*}\left[\xi_{i}\left(\theta_{0}\right)\left(z_{i 1}-z_{i 2}\right)^{\prime} \mid \Delta_{i}\right] f^{*}\left(\Delta_{i}\right)$,

$$
\mathcal{E}^{*}\left[\frac{\xi_{i}\left(\theta_{0}\right)\left(z_{i 1}-z_{i 2}\right)^{\prime}}{\varsigma^{2}} \kappa^{\prime}\left(\frac{\Delta_{i}}{\varsigma}\right)\right]=\int_{-\infty}^{+\infty} \frac{H(\Delta)}{\varsigma^{2}} \kappa^{\prime}\left(\frac{\Delta}{\varsigma}\right) \mathrm{d} \Delta
$$

which, by a change-of-variable argument, can be shown to converge to $\partial H(\Delta) /\left.\partial \Delta\right|_{\Delta=0}$, which is $O(1)$. Because $\sqrt{n \varsigma}\left(\gamma_{n}-\gamma_{0}\right)=o_{P}(1)$, this implies that the first term in (B.2) is $o_{P}(1 / \sqrt{n \varsigma})$. Similarly, the second term is bounded by

$$
\frac{\sup _{\varepsilon}\left|\kappa^{\prime \prime}(\varepsilon)\right| \frac{1}{n} \sum_{i=1}^{n} s_{i}\left\|\xi_{i}\left(\theta_{0}\right)\right\|\left\|z_{i 1}-z_{i 2}\right\|^{2}}{\varsigma^{3}}\left\|\gamma_{n}-\gamma_{0}\right\|^{2}=O_{P}\left(n^{3 r}\right) O_{P}\left(n^{-2 s}\right)=o_{P}\left(\frac{1}{\sqrt{n \varsigma}}\right)
$$

and so, too, can be ignored asymptotically. This establishes (B.1).

Asymptotic behavior of the infeasible moment. We next show that $\sqrt{n \varsigma} q_{n}\left(\theta_{0}\right) \stackrel{L}{\rightarrow} \mathcal{N}(0, \Sigma)$. Add and subtract $\mathcal{E}\left[g_{n}\left(\theta_{0}\right)\right]$ to write

$$
\sqrt{n \varsigma} q_{n}\left(\theta_{0}\right)=\sqrt{n \varsigma} \mathcal{E}\left[q_{n}\left(\theta_{0}\right)\right]+\sqrt{n \varsigma}\left\{q_{n}\left(\theta_{0}\right)-\mathcal{E}\left[q_{n}\left(\theta_{0}\right)\right]\right\} .
$$

The first term constitutes bias and is asymptotically negligible. Indeed,

$$
\left\|\mathcal{E}\left[q_{n}\left(\theta_{0}\right)\right]\right\|=\left\|\int_{-\infty}^{+\infty} \frac{\zeta\left(\Delta ; \theta_{0}\right)}{\varsigma} \kappa\left(\frac{\Delta_{i}}{\varsigma}\right) \mathrm{d} \Delta\right\| \leq \varsigma^{k} \frac{\sup _{\Delta}\left\|\nabla_{k} \zeta\left(\Delta ; \theta_{0}\right)\right\| \int_{-\infty}^{+\infty}\left|\eta^{k}\right||\kappa(\eta)| \mathrm{d} \eta}{k !} .
$$


Because the constant is bounded by Assumptions 3.3 and 3.5, $\sqrt{n \varsigma} \mathcal{E}\left[q_{n}\left(\theta_{0}\right)\right]=\sqrt{n \varsigma} O\left(\varsigma^{k}\right)$, which is $o(1)$ as we require that $r>\frac{1}{1+2 k}$.

The second term in (B.3) is the dominant term. To deal with it we verify the conditions for Lyapunov's central limit theorem for triangular arrays. Write

$$
\sqrt{n \varsigma}\left\{q_{n}\left(\theta_{0}\right)-\mathcal{E}\left[q_{n}\left(\theta_{0}\right)\right]\right\}=\frac{1}{\sqrt{n}} \sum_{i=1}^{n}\left\{\sigma_{i}-\mathcal{E}\left[\sigma_{i}\right]\right\}, \sigma_{i} \equiv \frac{\xi_{i}\left(\theta_{0}\right)}{\sqrt{\varsigma}} \kappa\left(\frac{\Delta_{i}}{\varsigma}\right) s_{i} .
$$

Then it suffices to show that (i) $\operatorname{var}\left[\sigma_{i}\right]<+\infty$ and $\lim _{n \uparrow+\infty} \operatorname{var}\left[\sigma_{i}\right]=\Sigma$, and that (ii) $\sum_{i=1}^{n} \mathcal{E}\left[\left\|\sigma_{i} / \sqrt{n}\right\|^{3}\right]=o(1)$. To show (i), recall that $\operatorname{var}\left[\sigma_{i}\right]=\mathcal{E}\left[\sigma_{i} \sigma_{i}^{\prime}\right]-\mathcal{E}\left[\sigma_{i}\right] \mathcal{E}\left[\sigma_{i}^{\prime}\right]$, and that

$$
\mathcal{E}\left[\sigma_{i}\right]=\sqrt{\varsigma} \int_{-\infty}^{+\infty} \frac{\zeta\left(\Delta ; \theta_{0}\right)}{\varsigma} \kappa\left(\frac{\Delta}{\varsigma}\right) \mathrm{d} \Delta, \quad \mathcal{E}\left[\sigma_{i} \sigma_{i}^{\prime}\right]=\int_{-\infty}^{+\infty} \frac{\Sigma\left(\Delta ; \theta_{0}\right)}{\varsigma} \kappa\left(\frac{\Delta}{\varsigma}\right)^{2} \mathrm{~d} \Delta .
$$

From above, $\mathcal{E}\left[\sigma_{i}\right]$ exists and is $o(1)$, while

$$
\mathcal{E}\left[\sigma_{i} \sigma_{i}^{\prime}\right]=\int_{-\infty}^{+\infty} \Sigma\left(\varsigma \eta ; \theta_{0}\right) \kappa(\eta)^{2} \mathrm{~d} \eta \rightarrow \Sigma\left(0 ; \theta_{0}\right) \int \kappa(\eta)^{2} \mathrm{~d} \eta=\Sigma
$$

by bounded convergence because $\sup _{\Delta}\left\|\Sigma\left(\Delta ; \theta_{0}\right)\right\|<+\infty$ and $\Sigma\left(\Delta ; \theta_{0}\right)$ is continuous in $\Delta$ in a neighborhood of zero. Thus, (i) is satisfied. To verify (ii), observe that

$$
\sum_{i=1}^{n} \mathcal{E}\left[\left\|\frac{\sigma_{i}}{\sqrt{n}}\right\|^{3}\right]=\sum_{i=1}^{n} \mathcal{E}\left[\left\|\frac{\xi_{i}\left(\theta_{0}\right)}{\sqrt{n \varsigma}} \kappa\left(\frac{\Delta_{i}}{\varsigma}\right)\right\|^{3}\right] \leq \frac{1}{\sqrt{n \varsigma}} \int_{-\infty}^{+\infty} \frac{g(\Delta)}{\varsigma}\left|\kappa\left(\frac{\Delta}{\varsigma}\right)\right|^{3} \mathrm{~d} \Delta,
$$

which is $O(1 / \sqrt{n \varsigma})$ because $g\left(\Delta_{i}\right) \equiv \mathcal{E}^{*}\left[\left\|\xi_{i}\left(\theta_{0}\right)\right\|^{3} \mid \Delta_{i}\right] f^{*}\left(\Delta_{i}\right) \operatorname{Pr}\left[s_{i}=1\right]$ is bounded. Hence, (ii) holds and

$$
\sqrt{n \varsigma} q_{n}\left(\theta_{0}\right) \stackrel{L}{\rightarrow} \mathcal{N}(0, \Sigma)
$$

has been shown.

Convergence of the Jacobian matrix. We use the same approach as used when establishing consistency. Fix $\theta \in \Theta$. An expansion gives

$$
\left\|\widehat{Q}_{n}(\theta)-Q_{n}(\theta)\right\| \leq \frac{\sup _{\varepsilon}\left|\kappa^{\prime}(\varepsilon)\right| \frac{1}{n} \sum_{i=1}^{n} s_{i}\left\|\xi_{i}^{\prime}(\theta)\right\|\left\|z_{i 1}-z_{i 2}\right\|}{\varsigma^{2}}\left\|\gamma_{n}-\gamma_{0}\right\|=o_{P}(1) .
$$

Also, $\left\|Q_{n}(\theta)-\mathcal{E}\left[Q_{n}(\theta)\right]\right\|=o_{P}(1)$ by the law of large numbers, and

$$
\mathcal{E}\left[Q_{n}(\theta)\right]=\int_{-\infty}^{+\infty} \frac{\zeta^{\prime}(\Delta ; \theta)}{\varsigma} \kappa\left(\frac{\Delta}{\varsigma}\right) \mathrm{d} \Delta \rightarrow Q_{0}(\theta)
$$

by bounded convergence. Pointwise convergence of the Jacobian has been established. Uniform convergence, i.e.,

$$
\sup _{\theta \in \Theta}\left\|\widehat{Q}_{n}(\theta)-Q_{0}(\theta)\right\|=o_{P}(1)
$$

follows from Lemma 2.9 in Newey and McFadden (1994). 
Asymptotic distribution. Using the results obtained so far the asymptotic distribution of $\theta_{n}$ is readily established. An expansion of the first-order conditions to the GMM minimization problem gives

$$
\sqrt{n \varsigma}\left(\theta_{n}-\theta_{0}\right)=-\left(Q_{0}^{\prime} V_{0} Q_{0}\right)^{-1} Q_{0}^{\prime} V_{0} \sqrt{n \varsigma} q_{n}\left(\theta_{0}\right)+o_{P}(1)
$$

on invoking $\left\|\theta_{n}-\theta_{0}\right\|=o_{P}(1)$ and appealing to (B.1)-(B.5). The delta method then yields

$$
\sqrt{n \varsigma}\left(\theta_{n}-\theta_{0}\right) \stackrel{L}{\rightarrow} \mathcal{N}(0, \Upsilon)
$$

where $\Upsilon=\left(Q_{0}^{\prime} V_{0} Q_{0}\right)^{-1}\left(Q_{0}^{\prime} V_{0} \Sigma V_{0} Q_{0}\right)\left(Q_{0}^{\prime} V_{0} Q_{0}\right)^{-1}$ for a generic positive definite $V_{0}$, and $\Upsilon=\left(Q_{0}^{\prime} \Sigma^{-1} Q_{0}\right)^{-1}$ for the optimally-chosen $V_{0}$.

Inference. Given that $V_{n} \stackrel{P}{\rightarrow} V_{0}$ by construction, it suffices to consider consistency of $Q_{n}$ and $\Sigma_{n} . Q_{n} \stackrel{P}{\rightarrow} Q_{0}$ follows from $\left\|\theta_{n}-\theta_{0}\right\|=o_{P}(1)$ and $\sup _{\theta \in \Theta}\left\|\widehat{Q}_{n}(\theta)-Q_{0}(\theta)\right\|=o_{P}(1)$, because $Q_{n}=\widehat{Q}_{n}\left(\theta_{n}\right)$. The argument for $\Sigma_{n} \stackrel{P}{\rightarrow} \Sigma$ is similar and is omitted.

\section{References}

Ahn, H. and J. L. Powell (1993). Semiparametric estimation of censored selection models with a nonparametric selection mechanism. Journal of Econometrics 58, 3-29.

Andrews, D. W. and M. M. A. Schafgans (1998). Semiparametric estimation of the intercept of a sample selection model. Review of Economic Studies 65, 497-517.

Arellano, M. and B. E. Honoré (2001). Panel data models: Some recent developments. In J. J. Heckman and E. Leamer (Eds.), Handbook of Econometrics, Volume V, Chapter 53, pp. 3229-3329. Elsevier.

Blundell, R. W. and J. L. Powell (2004). Endogeneity in semiparametric binary response models. Review of Economic Studies 71, 655-679.

Cameron, A. C. and P. K. Trivedi (2006). Regression Analysis of Count Data. Econometric Society Monographs. Cambridge University Press.

Chamberlain, G. (1992). Comment: Sequential moment restrictions in panel data. Journal of Business and Economic Statistics 10, 20-26.

Chamberlain, G. (2010). Binary response models for panel data: Identification and information. Econometrica 78, 159-168.

Charlier, E., B. Melenberg, and A. H. O. van Soest (1995). A smoothed maximum score estimator for the binary choice panel data model with an application to labour force participation. Statistica Nederlandica 49, 324-342. 
Domínguez, M. A. and I. N. Lobato (2004). Consistent estimation of models defined by conditional moment restrictions. Econometrica 72, 1601-1615.

Fernández-Val, I. and F. Vella (2011). Bias corrections for two-step fixed effects panel data estimators. Journal of Econometrics 163, 144-162.

Greene, W. (2009). Models for count data with endogenous participation. Empirical Economics 36, 133-173.

Gronau, R. (1973). The intrafamily allocation of time: The value of housewives' time. American Economic Review 63, 634-651.

Hall, A. R. (2005). Generalized Method of Moments. Advanced Texts in Econometrics. Oxford University Press.

Han, A. K. (1987). Non-parametric analysis of a generalized regression model: The maximum rank correlation estimator. Journal of Econometrics 35, 303-316.

Hansen, B. E. (2008). Uniform convergence rates for kernel estimation with dependent data. Econometric Theory 24, 726-748.

Hansen, L. P. (1982). Large sample properties of generalized method of moments estimators. Econometrica 50, 1029-1054.

Hansen, L. P., J. Heaton, and A. Yaron (1996). Finite-sample properties of some alternative GMM estimators. Journal of Business and Economic Statistics 14, 262-280.

Härdle, W., P. Hall, and H. Ichimura (1993). Optimal smoothing in single-index models. Annals of Statistics 21, 157-178.

Heckman, J. J. (1974). Shadow prices, market wages, and labor supply. Econometrica 42, 679-694.

Heckman, J. J. (1978). Dummy endogeneous variables in a simultaneous equation system. Econometrica 46, 931-959.

Heckman, J. J. (1979). Sample selection bias as a specification error. Econometrica 47, $153-161$.

Honoré, B. E. and E. Kyriazidou (2000). Panel data discrete choice models with lagged dependent variables. Econometrica 68, 839-874.

Horowitz, J. L. (1992). A smoothed maximum score estimator for the binary response model. Econometrica 60, 505-531.

Kleibergen, F. and R. Paap (2006). Generalized reduced rank tests using the singular value decomposition. Journal of Econometrics 133, 97-126. 
Kyriazidou, E. (1997). Estimation of a panel data sample selection model. Econometrica 65, $1335-1364$.

Kyriazidou, E. (2001). Estimation of dynamic panel data sample selection models. Review of Economic Studies 68, 543-572.

Lancaster, T. (2000). The incidental parameter problem since 1948. Journal of Econometrics 95, 391-413.

Lee, S. (2007). Endogeneity in quantile regression models: A control function approach. Journal of Econometrics 141, 1131-1158.

Li, Q. and J. S. Racine (2007). Nonparametric Econometrics: Theory and Practice. Princeton University Press.

Manski, C. F. (1975). Maximum score estimation of the stochastic utility model of choice. Journal of Econometrics 3, 205-228.

Manski, C. F. (1985). Semiparametric analysis of discrete response: Asymptotic properties of the maximum score estimator. Journal of Econometrics 27, 313-333.

Manski, C. F. (1987). Semiparametric analysis of random effects linear models from binary panel data. Econometrica 55, 357-362.

Müller, H.-G. (1984). Smooth optimum kernel estimators of densities, regression curves and modes. Annals of Statistics 12, 766-774.

Newey, W. K. (2009). Two-step series estimation of sample selection models. Econometrics Journal 12, S217-S229.

Newey, W. K. and D. L. McFadden (1994). Large sample estimation and hypothesis testing. In R. Engle and D. L. McFadden (Eds.), Handbook of Econometrics, Volume 4, Chapter 36, pp. 2111-2245. Elsevier.

Pakes, A. and D. Pollard (1989). Simulation and the asymptotics of optimization estimators. Econometrica 5\%, 1027-1057.

Powell, J. L. (1987). Semiparametric estimation of bivariate latent variable models. Working paper No. 8704, Social Systems Research Institute, University of Wisconsin-Madison.

Powell, J. L. (1994). Estimation of semiparametric models. In R. F. Engle and D. L. McFadden (Eds.), Handbook of Econometrics, Volume IV, Chapter 41, pp. 2443-2521. Elsevier.

Powell, J. L., J. H. Stock, and T. M. Stoker (1989). Semiparametric estimation of index coefficients. Econometrica 5\%, 1403-1430. 
Robinson, P. M. (1988). Root- $N$-consistent semiparametric regression. Econometrica 56, 931-954.

Rochina-Barrachina, M. E. (2008). A new estimator for panel data sample selection models. Annales d'Economie et de Statistique 55/56, 153-181.

Sargan, J. D. (1958). The estimation of economic relationships using instrumental variables. Econometrica 26, 393-415.

Sherman, R. P. (1993). The limiting distribution of the maximum rank correlation estimator. Econometrica 61, 123-137.

Stinchcombe, M. and H. White (1998). Consistent specification testing with nuisance parameters present only under the alternative. Econometric Theory 14, 295-325.

Terza, J. V. (1998). Estimating count data models with endogenous switching: Sample selection and endogenous treatment effects. Journal of Econometrics 84, 129-154.

Verbeek, M. and T. Nijman (1992). Testing for selectivity bias in panel data models. International Economic Review 33, 681-703.

Winkelmann, R. (1998). Count data models with selectivity. Econometric Reviews 17, 339-360.

Wooldridge, J. M. (1995). Selection corrections for panel data models under conditional mean independence assumptions. Journal of Econometrics 68, 115-132.

Wooldridge, J. M. (1997). Multiplicative panel data models without the strict exogeneity assumption. Econometric Theory 13, 667-678. 